\title{
Modified Sobolev Spaces in Controllability Problems for the Wave Equation on a Half-Plane
}

\author{
L.V. Fardigola \\ B. Verkin Institute for Low Temperature Physics and Engineering \\ National Academy of Sciences of Ukraine \\ 47 Lenin Ave., Kharkiv 61103, Ukraine \\ E-mail: fardigola@ukr.net, fardigola@ilt.kharkov.ua
}

Received November 26, 2013, revised October 15, 2014

\begin{abstract}
The 2-d wave equation $w_{t t}=\Delta w, t \in(0, T)$, on the half-plane $x_{1}>0$ controlled by the Neumann boundary condition $w_{x_{1}}\left(0, x_{2}, t\right)=\delta\left(x_{2}\right) u(t)$ is considered in Sobolev spaces, where $T>0$ is a constant and $u \in L^{\infty}(0, T)$ is a control. This control system is transformed into a control system for the 1-d wave equation in modified Sobolev spaces introduced and studied in the paper, and they play the main role in the study. The necessary and sufficient conditions of (approximate) $L^{\infty}$-controllability are obtained for the 1-d control problem. It is also proved that the 2-d control system replicates the controllability properties of the 1-d control system and vise versa. Finally, the necessary and sufficient conditions of (approximate) $L^{\infty}$ controllability are obtained for the 2-d control problem.
\end{abstract}

Key words: modified Sobolev space, wave equation, half-plane, controllability problem, Neumann boundary control.

Mathematics Subject Classification 2010: 93B05, 35B37, 35L05.

\section{Introduction}

Consider the wave equation on a half-plane

$$
w_{t t}=\Delta w, \quad x_{1}>0, x_{2} \in \mathbb{R}, t \in(0, T),
$$

controlled by the Neumann boundary condition

$$
w_{x_{1}}\left(0, x_{2}, t\right)=\delta\left(x_{2}\right) u(t), \quad x_{2} \in \mathbb{R}, t \in(0, T),
$$

where $T>0$, is a constant, $u \in L^{\infty}(0, T)$ is a control, $\delta$ is the Dirac distribution, $\Delta=\left(\partial / \partial x_{1}\right)^{2}+\left(\partial / \partial x_{2}\right)^{2}$. Control system (1.1), (1.2) is considered in the Sobolev spaces (see Secs. 2 and 3 ).

(c) L.V. Fardigola, 2015 
Note that most of the papers on controllability of the wave equation deal with bounded domains and consider $L^{p}$-controllability $(2 \leq p \leq+\infty)$ (see, e.g., $[8,11-$ $14,18]$ and others). Controllability problems for distributed parameter systems on domains unbounded with respect to the space variables have not been fully investigated. $L^{\infty}$-controllability and approximate $L^{\infty}$-controllability of the 1 -d wave equation on a half-axis were investigated at a given time and at a free time in $[4-7,15-17]$. Controllability problems for the 2 -d wave equation on a half-plane controlled by the Dirichlet boundary condition were studied only at a given time in the context of controls bounded by a hard constant in [3]. Controllability of the 3 -d wave equation in $\mathbb{R}^{3}$ were studied in [2]. In the present paper $L^{\infty}$ controllability and approximate $L^{\infty}$-controllability are considered at a given time and at a free time for the 2 -d wave equation on the half-plane $x_{1}>0$, where the equation is controlled by the Neumann boundary condition. In the case of the Neumann boundary control, the results on $L^{\infty}$-controllability at a given time are similar to those obtained in [3], where the equation controlled by the Dirichlet boundary condition was studied. However, the studying of the control problems at a given time differs from the studying of these problems at a free time. Thus, the methods used in the present paper essentially differ from those applied in [3]. We have to study some new spaces of the Sobolev type, the convergence in the spaces, and some operators acting in these spaces to investigate the controllability problems at a free time. Using these spaces and their properties, the necessary and sufficient conditions of (approximate) $L^{\infty}$-controllability are obtained at a given time and at a free time in the case of the Neumann boundary control.

The sketch of the paper is the following:

1. It is proved that control system (1.1), (1.2) under initial condition (2.1) is equivalent to control system (2.2), (2.3) (Sec. 2).

2. It is proved that if control system $(2.2),(2.3)$ is approximately $L^{\infty}$-controllable at a free time, then each solution to $(2.2),(2.3)$ is of the form $\mathrm{W}(x, t)=$ $\mathrm{w}(|x|, t)$ (Sec. 4), i.e., system (2.2), (2.3) is one-dimensional.

3. The operator $\boldsymbol{\Phi}$, transforming control system (2.2), (2.3) to auxiliary 1-d control system (4.1), (4.2), is introduced and studied (Sec. 3).

4. Applying the operator $\boldsymbol{\Phi}$ (see Sec. 3), it is proved that control system (2.2), (2.3) replicates the controllability properties of auxiliary control system (4.1), (4.2) and vice versa (Sec. 4).

5. Necessary and sufficient conditions of (approximate) $L^{\infty}$-controllability are obtained for auxiliary control system (4.1), (4.2) at a given time and at a free time (Sec. 5). 
6. Necessary and sufficient conditions of (approximate) $L^{\infty}$-controllability are obtained for the main control system (2.2), (2.3) at a given time and at a free time (Sec. 6).

The conditions of (approximate) $L^{\infty}$-controllability for the main control system (2.2), (2.3) are illustrated by the examples in Sec. 6 .

Note that auxiliary control system (4.1), (4.2) is considered in the modified Sobolev spaces that are the main objects as well as the main tools for studying the control problems in the present paper. There are two types of these spaces:

- the space $H_{s[1 / 2]}^{0}$ of the distributions $g \in \mathcal{S}^{\prime}$ such that we have $G \in H_{0}^{s}$ for $G(x)=g(|x|), s \in \mathbb{R}$;

- the space $H_{0}^{s[1 / 2]}$ that is the Fourier transform of $H_{s[1 / 2]}^{0}, s \in \mathbb{R}$.

These spaces are introduced and studied in Sec. 3. In particular, some embedding properties are proved for the pairs $H_{s[1 / 2]}^{0}, H_{s}^{0}$ and $H_{0}^{s[1 / 2]}, H_{0}^{s}, s \in \mathbb{R}$. The operator $\boldsymbol{\Phi}$ introduced and studied in the same section also plays an important role in the paper. This operator is an isomorphism of a subspace $\mathbb{H}_{0}^{s}$ of $H_{0}^{s}$ and $H_{0}^{s[1 / 2]}$ such that $\Delta \boldsymbol{\Phi} f=\boldsymbol{\Phi}\left(f^{\prime \prime}\right), f \in H_{0}^{s[1 / 2]}$. Here, $\mathbb{H}_{0}^{s}$ is the subspace of $H_{0}^{s}$ such that $F \in \mathbb{H}_{0}^{s}$ iff there exists $f \in H_{0}^{s[1 / 2]}$ under the condition $F(x)=f(|x|)$, $s \in \mathbb{R}$. Using the operator $\boldsymbol{\Phi}$, we can reduce control problem (1.1), (1.2), (2.1) to an auxiliary control problem for the 1-d wave equation (see (4.1), (4.2)). We should notice that control problem (4.1), (4.2) has been investigated in $H_{0}^{s}$ in [5]. However, we have to study this problem again because the convergence in the space $H_{0}^{s[1 / 2]}$ differs from that in $H_{0}^{s}$. Therefore the controls solving the (approximate) $L^{\infty}$-controllability problem for (4.1), (4.2) in $H_{0}^{s[1 / 2]}$ differ from those solving this problem in $H_{0}^{s}$. In particular, to construct the controls we have to prove Lemmas 7.1-7.7 that are rather complicated. Thus, using the operator $\boldsymbol{\Phi}$ and the modified Sobolev spaces $H_{s[1 / 2]}^{0}$ and $H_{0}^{s[1 / 2]}$, we solve the (approximate) $L^{\infty}$-controllability problem for control system (1.1), (1.2) at a given time and at a free time.

This study may be treated as an attempt to extend the class of operators transforming 1-d wave equation into more general equations (see, e.g., $[7,15,16]$ ). Note that the controllability problems for the wave equation with a variable potential were studied in [7] by reducing them to the controllability problems for the wave equation with a constant potential with the help of some transformation operator acting in the classical Sobolev spaces. In the present paper, we observe a similar transformation. The operator $\boldsymbol{\Phi}$ may be treated as a transformation operator transforming 2-d control problem (2.2), (2.3) in the classical Sobolev spaces into 1-d control problem (4.1), (4.2) in the modified Sobolev spaces. 


\section{Problem Formulation}

Let $n \in \mathbb{N}$. Let $\mathcal{S}\left(\mathbb{R}^{n}\right)$ be the Schwartz space of rapidly decreasing functions of $n$ variables and $\mathcal{S}^{\prime}\left(\mathbb{R}^{n}\right)$ be its dual space of tempered distributions (see, e.g., [10, Chap. 1]). Denote by $H_{l}^{s}\left(\mathbb{R}^{n}\right), s, l \in \mathbb{R}$, the following Sobolev spaces:

$$
\begin{aligned}
H_{l}^{s}\left(\mathbb{R}^{n}\right) & =\left\{\varphi \in \mathcal{S}\left(\mathbb{R}^{n}\right) \mid\left(1+|D|^{2}\right)^{s / 2}\left(1+|x|^{2}\right)^{l / 2} \varphi \in L^{2}\left(\mathbb{R}^{n}\right)\right\}, \\
\|\varphi\|_{l}^{s} & =\left(\int_{\mathbb{R}^{n}}\left|\left(1+|D|^{2}\right)^{s / 2}\left(1+|x|^{2}\right)^{l / 2} \varphi(x)\right|^{2} d x\right)^{1 / 2},
\end{aligned}
$$

where $|\cdot|$ is the Euclidian norm in $\mathbb{R}^{n}, D=\left(-i \partial / \partial x_{1}, \ldots,-i \partial / \partial x_{n}\right), n \in \mathbb{N}$. It is well known [10, Chap. 1] that $\|\varphi\|_{l^{\prime}}^{s^{\prime}} \leq\|\varphi\|_{l}^{s}, s^{\prime} \leq s, l^{\prime} \leq l, \varphi \in H_{l}^{s}\left(\mathbb{R}^{n}\right)$. Therefore, $H_{l}^{s} \subset H_{l^{\prime}}^{s^{\prime}}$ is a continuous embedding, $s^{\prime} \leq s, l^{\prime} \leq l$.

Let $\mathcal{F}: \mathcal{S}\left(\mathbb{R}^{n}\right) \rightarrow \mathcal{S}\left(\mathbb{R}^{n}\right)$ be the Fourier transform operator. For $\varphi \in \mathcal{S}\left(\mathbb{R}^{n}\right)$ we have $(\mathcal{F} \varphi)(\sigma)=(2 \pi)^{-n / 2} \int_{\mathbb{R}^{n}} e^{-i\langle x, \sigma\rangle} \varphi(x) d x$ and for $f \in \mathcal{S}^{\prime}\left(\mathbb{R}^{n}\right), \psi \in \mathcal{S}\left(\mathbb{R}^{n}\right)$, $\langle\mathcal{F} f, \psi\rangle=\left\langle f, \mathcal{F}^{-1} \psi\right\rangle$. It is well known [10, Chap. 1] that $\mathcal{F}$ is an isometric isomorphism of $H_{0}^{s}\left(\mathbb{R}^{n}\right)$ and $H_{s}^{0}\left(\mathbb{R}^{n}\right), s \in \mathbb{R}$. A distribution $f \in \mathcal{S}^{\prime}\left(\mathbb{R}^{n}\right)$ is said to be odd with respect to $x_{1}$ if $\left\langle f, \varphi\left(x_{1}, x_{2}, \ldots, x_{n}\right)\right\rangle=-\left\langle f, \varphi\left(-x_{1}, x_{2}, \ldots, x_{n}\right)\right\rangle, \varphi \in \mathcal{S}\left(\mathbb{R}^{n}\right)$, and be even with respect to $x_{1}$ if $\left\langle f, \varphi\left(x_{1}, x_{2}, \ldots, x_{n}\right)\right\rangle=\left\langle f, \varphi\left(-x_{1}, x_{2}, \ldots, x_{n}\right)\right\rangle$, $\varphi \in \mathcal{S}\left(\mathbb{R}^{n}\right)$.

Set $n=2, \mathbb{R}_{+}=(0,+\infty)$. For $s, l \in \mathbb{R}$, denote by $\widehat{H}_{l}^{s}\left(\mathbb{R}^{2}\right)$ the subspace of the distributions in $H_{l}^{s}\left(\mathbb{R}^{2}\right)$ that are even with respect to $x_{1}$, and denote $\widehat{\mathbf{H}}^{s}=\widehat{H}_{0}^{s}\left(\mathbb{R}^{2}\right) \times \widehat{H}_{0}^{s-1}\left(\mathbb{R}^{2}\right)$ and $\widehat{\mathbf{H}}_{l}=\widehat{H}_{l}^{0}\left(\mathbb{R}^{2}\right) \times \widehat{H}_{l-1}^{0}\left(\mathbb{R}^{2}\right)$, with the norms $\||\cdot|\|^{s}$ and $\|\mid \cdot\|_{l}$, respectively.

Set $s=0,1,2$. Denote also $\mathcal{H}_{0}^{s}=\left\{\varphi \in L^{2}\left(\mathbb{R}_{+} \times \mathbb{R}\right) \mid \exists \widehat{\varphi} \in \widehat{H}_{0}^{s}\left(\mathbb{R}^{2}\right) \varphi(x)=\right.$ $\widehat{\varphi}(x)$ a.e. on $\left.\mathbb{R}_{+} \times \mathbb{R}\right\}$ with the norm $\llbracket \varphi \rrbracket_{0}^{s}=\frac{1}{\sqrt{2}}\|\widehat{\varphi}\|_{0}^{s}, \varphi \in \mathcal{H}_{0}^{s}, \widehat{\varphi} \in \widehat{H}_{0}^{s}\left(\mathbb{R}^{2}\right)$, $\varphi(x)=\widehat{\varphi}(x)$ a.e. on $\mathbb{R}_{+} \times \mathbb{R}$, and $\mathcal{H}_{0}^{-s}=\left(\mathcal{H}_{0}^{s}\right)^{\prime}$ with the norm $\square f \rrbracket_{0}^{s}=$ $\sup \left\{|\langle f, \varphi\rangle| / \llbracket \varphi \rrbracket_{0}^{s} \mid \llbracket \varphi \rrbracket_{0}^{s} \neq 0\right\}, f \in \mathcal{H}_{0}^{-s}$. Evidently, for each $f \in \mathcal{H}_{0}^{-s}$ there exists the unique $\widehat{f} \in \widehat{H}_{0}^{-s}\left(\mathbb{R}^{2}\right)$ such that $\left.\widehat{f}\right|_{\mathbb{R}_{+} \times \mathbb{R}}=f$. Moreover, for each $\widehat{f} \in \widehat{H}_{0}^{-s}\left(\mathbb{R}^{2}\right)$ we have $f=\left.\widehat{f}\right|_{\mathbb{R}_{+} \times \mathbb{R}} \in \mathcal{H}_{0}^{-s}$. In addition, $\square f \rrbracket_{0}^{-s}=\frac{1}{\sqrt{2}}\|\widehat{f}\|_{0}^{-s}$ for $f \in \mathcal{H}_{0}^{-s}$ and $\widehat{f} \in \widehat{H}_{0}^{-s}\left(\mathbb{R}^{2}\right)$ such that $\left.\widehat{f}\right|_{\mathbb{R}_{+} \times \mathbb{R}}=f$. One can see that

$$
\begin{aligned}
& \mathcal{H}_{0}^{0}=L^{2}\left(\mathbb{R}_{+} \times \mathbb{R}\right), \quad \square \varphi \rrbracket_{0}^{0}=\|\varphi\|_{\mathcal{H}_{0}^{0}}, \varphi \in \mathcal{H}_{0}^{0} ; \\
& \mathcal{H}_{0}^{1}=\left\{\varphi \in \mathcal{H}_{0}^{0} \mid \varphi_{x_{1}} \in \mathcal{H}_{0}^{0} \text { and } \varphi_{x_{2}} \in \mathcal{H}_{0}^{0}\right\}, \\
& \square \varphi \rrbracket_{0}^{1}=\|\varphi\|_{\mathcal{H}_{0}^{0}}+\left\|\varphi_{x_{1}}\right\|_{\mathcal{H}_{0}^{0}}+\left\|\varphi_{x_{2}}\right\|_{\mathcal{H}_{0}^{0}}, \varphi \in \mathcal{H}_{0}^{1} ;
\end{aligned}
$$




$$
\begin{array}{r}
\mathcal{H}_{0}^{2}=\left\{\varphi \in \mathcal{H}_{0}^{1} \mid\left(\forall k, l=1,2, \varphi_{x_{k} x_{l}} \in \mathcal{H}_{0}^{0}\right) \text { and }\left(\varphi_{x_{1}}\left(+0, x_{2}\right)=0 \text { a.e. on } \mathbb{R}\right)\right\} \\
\square \varphi \rrbracket_{0}^{2}=\|\varphi\|_{\mathcal{H}_{0}^{0}}+2\left\|\varphi_{x_{1}}\right\|_{\mathcal{H}_{0}^{0}}+2\left\|\varphi_{x_{2}}\right\|_{\mathcal{H}_{0}^{0}}+2\left\|\varphi_{x_{1} x_{2}}\right\|_{\mathcal{H}_{0}^{0}} \\
+\left\|\varphi_{x_{1} x_{1}}\right\|_{\mathcal{H}_{0}^{0}}+\left\|\varphi_{x_{2} x_{2}}\right\|_{\mathcal{H}_{0}^{0}}, \varphi \in \mathcal{H}_{0}^{2}
\end{array}
$$

We treat equality (1.2) as the value of the distribution $w$ at $x_{1}=0$ (see Definition 2.1 below). Set $\mathcal{S}=\mathcal{S}(\mathbb{R}), \mathcal{S}_{+}=\left\{\varphi \in \mathcal{S} \mid \operatorname{supp} \varphi \in \mathbb{R}_{+}\right\}$, and $\mathcal{S}^{+}=$ $\left\{\varphi \in C^{\infty}\left(\mathbb{R}_{+}\right) \mid \forall k=\overline{0, \infty} \forall m=\overline{0, \infty} x^{k} \varphi^{(m)} \in L^{\infty}\left(\mathbb{R}_{+}\right)\right\}$. By analogy with the definition of the value of a distribution of one variable at a point [1, Chap. 1], we give the following definition for the value of a distribution of several variables at a line.

Definition 2.1. We say that a distribution $f \in\left(\mathcal{S}^{+}\right)^{\prime} \times \mathcal{S}^{\prime} \times\left(\mathcal{S}^{+}\right)^{\prime}$ has the value $f_{0} \in \mathcal{S}^{\prime} \times\left(\mathcal{S}^{+}\right)^{\prime}$ on the line $x_{1}=0$ (i.e., $f\left(0, x_{2}, x_{3}\right)=f_{0}\left(x_{2}, x_{3}\right)$ ) if for each $\varphi \in \mathcal{S}_{+} \times \mathcal{S} \times \mathcal{S}^{+}$we have $\left\langle f_{\alpha}-f_{0}, \varphi\right\rangle \rightarrow 0$ as $\alpha \rightarrow+0$, where $f_{\alpha}\left(x_{1}, x_{2}, x_{3}\right)=$ $f\left(\alpha x_{1}, x_{2}, x_{3}\right)$.

Consider control system (1.1),(1.2) under the initial condition

$$
w(x, 0)=w_{0}^{0}(x), \quad w_{t}(x, 0)=w_{1}^{0}, \quad x_{1}>0, x \in \mathbb{R},
$$

in the spaces $\mathcal{H}_{0}^{-s}, s=0,1,2$. Here $w_{0}^{0} \in \mathcal{H}_{0}^{1}, w_{1}^{0} \in \mathcal{H}_{0}^{0},\left(\frac{d}{d t}\right)^{s} w:[0, T] \rightarrow \mathcal{H}_{0}^{-s}$, $s=0,1,2, \Delta: \mathcal{H}_{0}^{0} \rightarrow \mathcal{H}_{0}^{-2}$. Denote by $\mathrm{W}(\cdot, t)=\left(\begin{array}{c}\mathrm{W}_{0}(\cdot, t) \\ \mathrm{W}_{1}(\cdot, t)\end{array}\right)$ and $\mathrm{W}^{0}=\left(\begin{array}{c}\mathrm{W}_{0}^{0} \\ \mathrm{~W}_{1}^{0}\end{array}\right)$ the even extension of $\left(\begin{array}{c}w \\ w_{t}\end{array}\right)$ and $\left(\begin{array}{c}w_{0}^{0} \\ w_{1}^{0}\end{array}\right)$, respectively, with respect to $x_{1}, t \in[0, T]$. Then, $\left(\frac{d}{d t}\right)^{s} \mathrm{~W}:[0, T] \rightarrow \widehat{\mathbf{H}}^{-s}, s=0,1, \mathrm{~W}^{0} \in \widehat{\mathbf{H}}^{1}$. If $w$ is a solution to problem (1.1), (2.1), (1.2), then $\mathrm{W}$ is a solution to problem

$$
\begin{aligned}
& \frac{d}{d t} \mathrm{~W}=\left(\begin{array}{ll}
0 & 1 \\
\Delta & 0
\end{array}\right) \mathrm{W}-2 \delta u(t)\left(\begin{array}{l}
0 \\
1
\end{array}\right), \quad t \in(0, T), \\
& \mathrm{W}(\cdot, 0)=\mathrm{W}^{0},
\end{aligned}
$$

where $\delta \in H_{0}^{-2}\left(\mathbb{R}^{2}\right)$ is the Dirac distribution with respect to $x$. Due to the Poisson formula (see, e.g., [19, Chap. 3]), we have

$$
\begin{aligned}
\mathrm{W}(x, t)= & \frac{1}{2 \pi} \frac{\partial}{\partial t}\left(\frac{H\left(t^{2}-|x|^{2}\right)}{\sqrt{t^{2}-|x|^{2}}} * \mathrm{~W}_{0}^{0}\right)+\frac{1}{2 \pi} \frac{H\left(t^{2}-|x|^{2}\right)}{\sqrt{t^{2}-|x|^{2}}} * \mathrm{~W}_{1}^{0} \\
& -\frac{1}{\pi} \int_{0}^{t} \frac{H\left(\xi^{2}-|x|^{2}\right)}{\sqrt{\xi^{2}-|x|^{2}}} u(t-\xi) d \xi
\end{aligned}
$$

where $*$ is the convolution with respect to $x$. Let $\mathrm{W}^{+}$be the restriction of $\mathrm{W}$ to $\mathbb{R}_{+} \times \mathbb{R} \times[0, T]$. Taking into account Lemmas 7.8 and 7.9 , we obtain

$$
\left(\mathrm{W}_{0}^{+}\right)_{x_{1}}\left(0, x_{2}, t\right)=\delta\left(x_{2}\right) u(t) .
$$


Therefore, if $\mathrm{W}$ is a solution to problem $(2.2),(2.3)$, then $\mathrm{W}^{+}$is a solution to problem (1.1), (2.1), (1.2). Thus, control problems (1.1), (2.1), (1.2) and (2.2), (2.3) are equivalent. Further we will consider control problem (2.2), (2.3) instead of control problem (1.1), (2.1), (1.2).

\section{Spaces and Operators}

Let us give definitions of the spaces used in the paper. Set $n=1$. For $s, l \in \mathbb{R}$ denote $H_{l}^{s}=H_{l}^{s}(\mathbb{R})$, and denote by $\widehat{H}_{l}^{s}$ the subspace of even distributions in $H_{l}^{s}$. Further throughout the section we will assume $s \in \mathbb{R}$.

Introduce the space $H_{s[-1 / 2]}^{0}=\left\{\varphi \in H_{s-1 / 2}^{0} \mid \exists \bar{\varphi} \in H_{s}^{0} \varphi=\sqrt{|\rho|} \bar{\varphi}\right\}$ with the norm $|\varphi|_{s[-1 / 2]}^{0}=\|\varphi / \sqrt{|\rho|}\|_{s}^{0}, \varphi \in H_{s[-1 / 2]}^{0}$, and its dual space $H_{-s[1 / 2]}^{0}=$ $\left(H_{s[-1 / 2]}^{0}\right)^{\prime}$ with the strong topology, i.e., $|f|_{-s[1 / 2]}^{0}=\sup \left\{|\langle f, \varphi\rangle| /|\varphi|_{s[-1 / 2]}^{0} \mid\right.$ $\left.|\varphi|_{s[-1 / 2]}^{0} \neq 0\right\}, f \in H_{-s[1 / 2]}^{0}$. Evidently, $|f|_{-s[1 / 2]}^{0}=\|\sqrt{|\rho|} f\|_{-s}^{0}, f \in H_{-s[1 / 2]}^{0}$. One can see that $H_{s[-1 / 2]}^{0}$ and $H_{-s[1 / 2]}^{0}$ are complete. Since $\sqrt{|\rho|} \leq \sqrt[4]{1+\rho^{2}}$, it is seen that

$$
\|\varphi\|_{s-1 / 2}^{0} \leq|\varphi|_{s[-1 / 2]}^{0}, \varphi \in H_{s[-1 / 2]}^{0} ;|f|_{-s[1 / 2]}^{0} \leq\|f\|_{-s+1 / 2}^{0}, f \in H_{-s+1 / 2}^{0} .
$$

Therefore, $H_{s[-1 / 2]}^{0} \subset H_{s-1 / 2}^{0}$ and $H_{-s+1 / 2}^{0} \subset H_{-s[1 / 2]}^{0}$ are continuous embeddings. According to Lemma 7.1, if $f \in H_{-s[1 / 2]}^{0}$, then $f \in H_{-s+1 / 2}^{-3 / 2}$.

Introduce the spaces $H_{0}^{s[-1 / 2]}=\mathcal{F} H_{s[-1 / 2]}^{0}$ and $H_{0}^{-s[1 / 2]}=\mathcal{F} H_{-s[1 / 2]}^{0}$ with the norms $|\varphi|_{0}^{s[-1 / 2]}=|\mathcal{F} \varphi|_{s[-1 / 2]}^{0}, \varphi \in H_{0}^{s[-1 / 2]} 0$ and $|f|_{0}^{-s[1 / 2]}=|\mathcal{F} f|_{-s[1 / 2]}^{0}$, $f \in H_{0}^{-s[1 / 2]}$. Evidently, $H_{0}^{-s[1 / 2]}=\left(H_{0}^{s[-1 / 2]}\right)^{\prime}$. One can see that $H_{0}^{s[-1 / 2]}$ and $H_{0}^{-s[1 / 2]}$ are complete. Taking into account (3.1), we get

$$
\|\varphi\|_{0}^{s-1 / 2} \leq|\varphi|_{0}^{s[-1 / 2]}, \varphi \in H_{0}^{s[-1 / 2]} ;|f|_{0}^{-s[1 / 2]} \leq\|f\|_{0}^{-s+1 / 2}, f \in H_{0}^{-s+1 / 2} .
$$

Therefore, $H_{0}^{s[-1 / 2]} \subset H_{0}^{s-1 / 2}$ and $H_{0}^{-s+1 / 2} \subset H_{0}^{-s[1 / 2]}$ are continuous embeddings. According to Lemma 7.1, if $f \in H_{0}^{-s[1 / 2]}$, then $f \in H_{-3 / 2}^{-s+1 / 2}$. For $f \in$ $H_{0}^{-s[1 / 2]}$, by analogy with [10, Chap. 1], setting $F=\mathcal{F} f$ and $f_{s}=\left(1+|D|^{2}\right)^{s / 2} f$, we obtain

$$
|f|_{0}^{-s[1 / 2]}=|F|_{-s[1 / 2]}^{0}=\left(\frac{1}{2 \pi} \int_{-\infty}^{\infty} \int_{-\infty}^{\infty}\left(1+\rho^{2}\right)^{-s}|F(\rho)|^{2} \frac{\left|e^{i z \rho}-1\right|^{2}}{z^{2}} d u d \rho\right)^{1 / 2}
$$




$$
\begin{aligned}
& =\left(\frac{1}{2 \pi} \iint_{\mathbb{R}^{2}} \frac{\left|f_{s}(x+z)-f_{s}(x)\right|^{2}}{|z|^{2}} d x d z\right)^{1 / 2} \\
& =\left(\frac{1}{2 \pi} \iint_{\mathbb{R}^{2}} \frac{\left|f_{s}(x)-f_{s}(y)\right|^{2}}{|x-y|^{2}} d y d x\right)^{1 / 2} .
\end{aligned}
$$

Denote by $\widehat{H}_{-s[1 / 2]}^{0}$ and $\widehat{H}_{0}^{-s[1 / 2]}$ the subspaces of even distributions in $H_{-s[1 / 2]}^{0}$ and $H_{0}^{-s[1 / 2]}$, respectively. Now set $n=2$ and introduce the subspaces

$$
\begin{aligned}
& \mathbb{H}_{0}^{s}=\left\{G \in H_{0}^{s}\left(\mathbb{R}^{2}\right) \mid \exists g \in \mathcal{S}_{+}^{\prime} G(x)=g(|x|)\right\}, \\
& \mathbb{H}_{s}^{0}=\left\{F \in H_{s}^{0}\left(\mathbb{R}^{2}\right) \mid \exists f \in \mathcal{S}_{+}^{\prime} F(x)=f(|x|)\right\}
\end{aligned}
$$

of $H_{0}^{s}\left(\mathbb{R}^{2}\right)$ and $H_{s}^{0}\left(\mathbb{R}^{2}\right)$, respectively. If $f \in \mathbb{H}_{s}^{0}$, then there exists the unique $f \in \widehat{H}_{s[1 / 2]}^{0}$ such that $F(x)=f(|x|), x \in \mathbb{R}^{2}$, and

$$
\|F\|_{s}^{0}=\sqrt{\pi}|f|_{s[1 / 2]}^{0} .
$$

Therefore, $\mathbb{H}_{s}^{0}$ is isomorphic to $\widehat{H}_{s}^{0[1 / 2]}$. Hence the space $\mathbb{H}_{s}^{0}$ is complete. Due to [10, Chap. 1], the Fourier transform $\mathcal{F}$ is an isometric isomorphism of $H_{0}^{s}\left(\mathbb{R}^{2}\right)$ and $H_{s}^{0}\left(\mathbb{R}^{2}\right)$. Therefore, $\mathcal{F}$ is an isometric isomorphism of $\mathbb{H}_{0}^{s}$ and $\mathbb{H}_{s}^{0}$. Thus $\mathbb{H}_{0}^{s}$ is also complete.

Let $\widehat{H}_{0[1 / 2]}^{s}$ and $\mathbb{I}_{s}^{0}: \mathbb{H}_{s}^{0} \rightarrow \widehat{H}_{s[1 / 2]}^{0}$ be the isomorphisms of $\mathbb{H}_{s}^{0}$ and $\widehat{H}_{s[1 / 2]}^{0}$ mentioned above. We have $\mathbb{I}_{s}^{0} F=f$ iff $F(x)=f(|x|), x \in \mathbb{R}^{2}$.

Denote $\boldsymbol{\Phi}: \widehat{H}_{0}^{-2[1 / 2]} \rightarrow \mathbb{H}_{0}^{-2}, D(\boldsymbol{\Phi})=\widehat{H}_{0}^{-2[1 / 2]}$,

$$
\mathbf{\Phi} f=\left(\mathcal{F}^{-1}\left(\mathbb{I}_{-2}^{0}\right)^{-1} \mathcal{F}\right) f, \quad f \in D(\boldsymbol{\Phi}) .
$$

Taking into account (3.4), we obtain

Theorem 3.1. $\boldsymbol{\Phi}$ is an isomorphism of $\widehat{H}_{0}^{s[1 / 2]}$ and $\mathbb{H}_{0}^{s}$. In addition, $\|\mathbf{\Phi} f\|_{0}^{s}=$ $\sqrt{\pi}|f|_{0}^{-s[1 / 2]}, f \in D(\boldsymbol{\Phi}), s \geq-2$.

We also need

Theorem 3.2. We have $\Delta \boldsymbol{\Phi} f=\boldsymbol{\Phi}\left(f^{\prime \prime}\right), f \in \widehat{H}_{0}^{s[1 / 2]}, s \geq-2$.

P r o o f. For $f \in \widehat{H}_{0}^{s[1 / 2]}$, we have

$$
\Delta \boldsymbol{\Phi} f=-\mathcal{F}^{-1}\left(|\sigma|^{2}\left(\left(\mathbb{I}_{-2}^{0}\right)^{-1} \mathcal{F} f\right)\right)=-\left(\mathcal{F}^{-1}\left(\mathbb{I}_{-2}^{0}\right)^{-1}\right)\left(\rho^{2} \mathcal{F} f\right)=\boldsymbol{\Phi}\left(f^{\prime \prime}\right) .
$$

That was to be proved. 
Theorem 3.3. Let $\alpha>0, f \in \widehat{H}_{0}^{0[1 / 2]}$, and $G=\boldsymbol{\Phi} f$. Then, $\operatorname{supp} f \subset[-\alpha, \alpha]$ iff $\operatorname{supp} G \subset D_{\alpha}=\left\{x \in \mathbb{R}^{2}|| x \mid \leq \alpha\right\}$.

P r o o f. Due to Theorem $3.1, G \in \mathbb{H}_{0}^{0}$. Put $F=\mathcal{F} f$ and $g=\mathcal{F} G$. Then,

$$
g(\sigma)=F(|\sigma|), \quad \sigma \in \mathbb{R} .
$$

First, let $\operatorname{supp} f \subset[-\alpha, \alpha]$. Due to Lemma 7.1, $f \in \widehat{H}_{-3 / 2}^{1 / 2} \subset \mathcal{S}^{\prime}$. Applying the generalized Paley-Wiener theorem [9, Chap. 3], we may conclude that $F$ can be extended to an entire function of the order $\leq 1$ and the type $\leq \alpha$. Since $G \in \mathbb{H}_{0}^{0} \subset L^{2}\left(\mathbb{R}^{2}\right)$ is even, we obtain that $g \in L^{2}\left(\mathbb{R}^{2}\right)$ and it can be extended to an entire function of the order $\leq 1$ and the type $\leq \alpha$. Applying the Paley-Wiener theorem, we obtain supp $G \subset[-\alpha, \alpha]^{2}$. Therefore, $\operatorname{supp} G \subset D_{\alpha}$ because $G \in \mathbb{H}_{0}^{0}$.

Now, let $\operatorname{supp} G \subset D_{\alpha}$. Then, $\operatorname{supp} G \subset[-\alpha, \alpha]^{2}$. Applying again the PaleyWiener theorem, we obtain that $g \in \mathbb{H}_{0}^{0}$ and it can be extended to an entire function of the order $\leq 1$ and the type $\leq \alpha$. Moreover,

$$
|g(s)| \leq \frac{\alpha}{2 \sqrt{\pi}}\|G\|_{0}^{0} e^{|\Im s|} \leq \frac{\alpha}{2 \sqrt{\pi}}\|G\|_{0}^{0} e^{|s|}, \quad s \in \mathbb{C},
$$

because $G \in \mathbb{H}_{0}^{0}$. Therefore $F$ is a regular distribution, $F$ is of a polynomial growth, and $F$ can be extended to an entire function of the order $\leq 1$ and the type $\leq \alpha$. Applying the generalized Paley-Wiener theorem [9, Chap. 3], we obtain supp $f \subset[-\alpha, \alpha]$. The theorem is proved.

Denote $\widehat{\mathbb{H}}^{s}=\mathbb{H}_{0}^{s} \times \mathbb{H}_{0}^{s-1}$ and $\widehat{\mathbb{H}}_{s}=\mathbb{H}_{s}^{0} \times \mathbb{H}_{s-1}^{0}$, and consider them as subspaces of $\widehat{\mathbf{H}}^{s}$ and $\widehat{\mathbf{H}}_{s}$, respectively.

One can see that the following two theorems hold.

Theorem 3.4. Let $f \in D(\boldsymbol{\Phi})$ and $f^{\prime} \in L^{\infty}(\mathbb{R}) \cap L^{1}(\mathbb{R})$. Then, $(\boldsymbol{\Phi} f)(x)=$ $\sqrt{\frac{2}{\pi}} \int_{|x|}^{\infty} \frac{f^{\prime}(\xi) d \xi}{\sqrt{\xi^{2}-|x|^{2}}}$

Theorem 3.5. Let $G \in D\left(\boldsymbol{\Phi}^{-1}\right), g=\mathbb{I}_{0}^{-2} G, r g \in L^{1}(\mathbb{R})$, and $r g \in L^{\infty}(-a, a)$ for each $a>0$. Then, $\left(\boldsymbol{\Phi}^{-1} G\right)(\xi)=\sqrt{\frac{2}{\pi}} \int_{\xi}^{\infty} \frac{r g(r) d r}{\sqrt{r^{2}-\xi^{2}}}$.

\section{Transformations between Two-Dimensional and One-Dimensional Control Systems}

Consider control system (2.2), (2.3) and the auxiliary control system

$$
\begin{aligned}
\frac{d}{d t} \mathrm{Z}(\cdot, t) & =\left(\begin{array}{cc}
0 & 1 \\
(d / d \xi)^{2} & 0
\end{array}\right) \mathrm{Z}(\cdot, t)+\left(\begin{array}{c}
0 \\
1
\end{array}\right) \sqrt{\frac{2}{\pi}} \delta u(t), \quad t \in(0, T), \\
\mathrm{Z}(\cdot, 0) & =\mathrm{Z}^{0}
\end{aligned}
$$


with the same time $T>0$ and the same control $u \in L^{\infty}(0, T)$. Here $\delta \in H_{0}^{-2[1 / 2]}$ is the Dirac distribution with respect to $\xi,\left(\frac{d}{d t}\right)^{s} \mathrm{Z}:[0, T] \rightarrow \widehat{\mathbf{H}}^{-s[1 / 2]}, s=0,1$, $\mathrm{Z}^{0} \in \widehat{\mathbf{H}}^{1[1 / 2]}$.

For given $T>0$ and $\mathrm{W}^{0} \in \widehat{\mathbf{H}}^{1}\left(\mathrm{Z}^{0} \in \widehat{\mathbf{H}}^{1[1 / 2]}\right)$, denote by $\mathcal{R}_{T}^{2}\left(\mathrm{~W}^{0}\right)\left(\mathcal{R}_{T}^{1}\left(\mathrm{Z}^{0}\right)\right.$, respectively) the set of the states $\mathrm{W}^{T} \in \widehat{\mathbf{H}}^{1}$ ( $\mathrm{Z}^{T} \in \widehat{\mathbf{H}}^{1[1 / 2]}$, respectively) for which there exists a control $u \in L^{\infty}(0, T)$ such that problem (2.2), (2.3) ((4.1), (4.2), respectively) has the unique solution $\mathrm{W}$ (Z, respectively) and $\mathrm{W}(\cdot, T)=\mathrm{W}^{T}$ $\left(\mathrm{Z}(\cdot, T)=\mathrm{Z}^{T}\right.$, respectively). Denote also $\mathcal{R}_{\infty}^{j}\left(\mathrm{Z}^{0}\right)=\bigcup_{T>0} \mathcal{R}_{T}^{j}\left(\mathrm{Z}^{0}\right), j=1,2$.

Definition 4.1. A state $\mathrm{W}^{0} \in \widehat{\mathbf{H}}^{1}\left(\mathrm{Z}^{0} \in \widehat{\mathbf{H}}^{1[1 / 2]}\right)$ is called $L^{\infty}$-controllable with respect to system (2.2), (2.3) ((4.1), (4.2), respectively) at a given time $T>0$ if 0 belongs to $\mathcal{R}_{T}^{2}\left(\mathrm{~W}^{0}\right)\left(\mathcal{R}_{T}^{1}\left(\mathrm{Z}^{0}\right)\right.$, respectively) and approximately $L^{\infty}$ controllable with respect to this system at a given time $T>0$ if 0 belongs to the closure of $\mathcal{R}_{T}^{2}\left(\mathrm{Z}^{0}\right)$ in $\widehat{\mathbf{H}}^{1}$ (the closure of $\mathcal{R}_{T}^{1}\left(\mathrm{Z}^{0}\right)$ in $\widehat{\mathbf{H}}^{1[1 / 2]}$, respectively).

Definition 4.2. A state $\mathrm{W}^{0} \in \widehat{\mathbf{H}}^{1}\left(\mathrm{Z}^{0} \in \widehat{\mathbf{H}}^{1[1 / 2]}\right)$ is called approximately $L^{\infty}$ controllable with respect to system (2.2), (2.3) ((4.1), (4.2), respectively) at a free time if 0 belongs to the closure of $\mathcal{R}_{T}^{2}\left(\mathrm{Z}^{0}\right)$ in $\widehat{\mathbf{H}}^{1}$ (the closure of $\mathcal{R}_{T}^{1}\left(\mathrm{Z}^{0}\right)$ in $\widehat{\mathbf{H}}^{1[1 / 2]}$, respectively).

Theorem 4.1. Let $u_{n}(t), t \in\left[0, T_{n}\right], n=\overline{1, \infty}$, solve the approximate $L^{\infty}$ controllability problem with respect to system (2.2), (2.3) for a state $\mathrm{W}^{0} \in \widehat{\mathbf{H}}^{1}$. Let $\mathrm{W}^{n}$ be a solution to (2.2), (2.3) with $u=u_{n}, T=T_{n}, n=\overline{1, \infty}$. Then, this solution is unique, $\mathrm{W}^{0} \in \widehat{\mathbb{H}}^{1}, \mathrm{~W}^{n}(\cdot, t) \in \widehat{\mathbb{H}}^{0}, t \in\left[0, T_{n}\right], n=\overline{1, \infty}$.

P r o o f. Since the controls $u_{n}(t), t \in\left[0, T_{n}\right], n=\overline{1, \infty}$, solve the approximate $L^{\infty}$-controllability problem with respect to system $(2.2),(2.3)$ for the state $\mathrm{W}^{0} \in$ $\widehat{\mathbf{H}}^{1}$, we have

$$
\left\|\mathrm{W}^{n}\left(\cdot, T_{n}\right)\right\|^{1} \rightarrow 0 \quad \text { as } n \rightarrow \infty .
$$

Put $\mathrm{V}^{0}=\mathcal{F W}^{0}, \mathrm{~V}^{n}(\cdot, t)=\mathcal{F} \mathrm{W}^{n}(\cdot, t), t \in\left[0, T_{n}\right], n=\overline{1, \infty}$. For $n=\overline{1, \infty}$, applying the Fourier transform with respect to $x$ to system $(2.2),(2.3)$ with $u=u_{n}, T=T_{n}$, we obtain

$$
\begin{aligned}
& \frac{d}{d t} \mathrm{~V}^{n}=\left(\begin{array}{cc}
0 & 1 \\
-|\sigma|^{2} & 0
\end{array}\right) \mathrm{V}^{n}-\frac{1}{\pi} u_{n}(t)\left(\begin{array}{l}
0 \\
1
\end{array}\right), \quad t \in(0, T), \\
& \mathrm{V}(\cdot, 0)=\mathrm{V}^{0},
\end{aligned}
$$

where $\left(\frac{d}{d t}\right)^{s} \mathrm{~V}:[0, T] \rightarrow \widehat{\mathbf{H}}_{-s}, s=0,1, \mathrm{~V}^{0} \in \widehat{\mathbf{H}}_{1}$. Hence, for $n=\overline{1, \infty}$, we have that

$$
\mathrm{V}^{n}(\sigma, t)=\left(\begin{array}{cc}
\cos (t|\sigma|) & \frac{\sin (t|\sigma|)}{|\sigma|} \\
-|\sigma| \sin (t|\sigma|) & \cos (t|\sigma|)
\end{array}\right)\left(\mathrm{V}^{0}(\sigma)-\frac{1}{\pi} \int_{0}^{t}\left(\begin{array}{c}
\frac{\sin (\xi|\sigma|)}{|\sigma|} \\
\cos (\xi|\sigma|)
\end{array}\right) u_{n}(\xi) d \xi\right)
$$


is the unique solution to (4.4), (4.5). Thus $\mathrm{W}$ is the unique solution to (2.2), (2.3). Taking into account (4.3), we get

$$
\left\|\mathrm{V}^{n}\left(\cdot, T_{n}\right)\right\|^{1} \rightarrow 0 \quad \text { as } n \rightarrow \infty .
$$

Set $\mathcal{U}_{n}(t)=u_{n}(t)(H(t)-H(t-T))-u_{n}(-t)(H(t+T)-H(t)), t \in \mathbb{R} ; \nu_{n}=\mathcal{F U}_{n} ;$ $\tilde{\nu}_{n}(\sigma)=\nu_{n}(|\sigma|)-\nu_{n}(|\sigma|), \hat{\nu}_{n}(\sigma)=\nu_{n}(|\sigma|)+\nu_{n}(|\sigma|), \sigma \in \mathbb{R}^{2}, n=\overline{1, \infty}$. Taking into account (4.7), we get

$$
\left\||\sigma| \mathrm{V}_{0}^{0}-\sqrt{\frac{2}{\pi}} \tilde{\nu}_{n}\right\|_{0}^{0} \rightarrow 0 \quad \text { and } \quad\left\|\mathrm{V}_{1}^{0}-\sqrt{\frac{2}{\pi}} \hat{\nu}_{n}\right\|_{0}^{0} \rightarrow 0 \quad \text { as } n \rightarrow \infty .
$$

Since $\mathbb{H}_{1}^{0}$ is complete and $\mathrm{V}^{0} \in \widehat{\mathbf{H}}_{1}$, we have $\mathrm{V}^{0} \in \widehat{\mathbb{H}}_{1}$. Therefore, $\mathrm{W}^{0} \in \widehat{\mathbb{H}}^{1}$. With regard to $(4.6)$ we obtain $\mathrm{V}^{n}(\cdot, t) \in \widehat{\mathbb{H}}_{0}, t \in\left[0, T_{n}\right]$, and thus $\mathrm{W}^{n}(\cdot, t) \in \widehat{\mathbb{H}}^{0}$, $t \in\left[0, T_{n}\right], n=\overline{1, \infty}$. The theorem is proved.

Taking into account (4.7), we obtain

Corollary 4.2. Let $T>0, u \in L^{\infty}(0, T)$, and $\mathrm{W}^{0} \in \widehat{\mathbb{H}}^{1}$. Let $\mathrm{W}$ be a solution to $(2.2)$, (2.3). Then, $\mathrm{W}(\cdot, t) \in \widehat{\mathbb{H}}^{0}, t \in[0, T]$.

According to Theorem 4.1 and Corollary 4.2, we can consider the $L^{\infty}$-controllability problems with respect to system $(2.2),(2.3)$ in the spaces $\widehat{\mathbb{H}}^{s}$ instead of the spaces $\widehat{\mathbf{H}}^{s}, s=0,1$.

Theorem 4.2. Let $T>0, u \in L^{\infty}(0, T), \mathrm{Z}^{0} \in \widehat{\mathbf{H}}^{1[1 / 2]}, \mathrm{W}^{0}=\mathbf{\Phi} \mathrm{Z}^{0}$. Let $\mathrm{Z}$ be a solution to (4.1), (4.2) and $\mathrm{W}(\cdot, T)=\mathbf{\Phi Z}(\cdot, t), t \in[0, T]$. Then $\mathrm{W}$ is a solution to $(2.2),(2.3), \mathrm{W}^{0} \in \widehat{\mathbb{H}}^{1}$, and $\mathrm{W}(\cdot, t) \in \widehat{\mathbb{H}}^{0}, t \in[0, T]$.

P r o o f. We have

$$
\boldsymbol{\Phi} \delta=\mathcal{F}^{-1}\left(\mathbb{I}_{-2}^{0}\right)^{-1} \mathcal{F} \delta=\sqrt{2 \pi} .
$$

Taking into account Theorems 3.1, 3.2 and applying $\boldsymbol{\Phi}$ to (4.1), (4.2), we obtain (2.2), (2.3) for $\mathrm{W}^{0}=\boldsymbol{\Phi} \mathrm{Z}^{0}$ and $\mathrm{W}(\cdot, T)=\boldsymbol{\Phi Z}(\cdot, t), t \in[0, T]$. Hence $\mathrm{W}$ is a solution to $(2.2),(2.3)$. Moreover, $\mathrm{W}^{0} \in \widehat{\mathbb{H}}^{1}$, and $\mathrm{W}(\cdot, t) \in \widehat{\mathbb{H}}^{0}, t \in[0, T]$. The theorem is proved.

Theorem 4.3. Let $T>0, u \in L^{\infty}(0, T), \mathrm{W}^{0} \in \widehat{\mathbb{H}}^{1}, \mathrm{Z}^{0}=\mathbf{\Phi}^{-1} \mathrm{~W}^{0}$. Let $\mathrm{W}$ be a solution to $(2.2),(2.3)$ and $\mathrm{Z}(\cdot, T)=\boldsymbol{\Phi}^{-1} \mathrm{~W}(\cdot, t), t \in[0, T]$. Then, $\mathrm{Z}$ is a solution to (4.1), (4.2), $\mathrm{Z}^{0} \in \widehat{\mathbf{H}}^{1[1 / 2]}$, and $\mathrm{Z}(\cdot, t) \in \widehat{\mathbf{H}}^{1[1 / 2]}, t \in[0, T]$.

P r o o f. Taking into account (4.8), Theorems 3.1, 3.2 and applying $\boldsymbol{\Phi}^{-1}$ to $(2.2)$, (2.3), we obtain $4.1,4.2$ for $\mathrm{Z}^{0}=\boldsymbol{\Phi}^{-1} \mathrm{~W}^{0}$ and $\mathrm{Z}(\cdot, T)=\boldsymbol{\Phi}^{-1} \mathrm{~W}(\cdot, t)$, $t \in[0, T]$. Hence $\mathrm{Z}$ is a solution to (4.1), (4.2). That was to be proved. 
Theorems 3.1, 4.1-4.3 imply

Corollary 4.5. Let $\mathrm{W}^{0} \in \widehat{\mathbb{H}}^{1}$ and $\mathrm{Z}^{0}=\mathbf{\Phi}^{-1} \mathrm{~W}^{0}$. Then the following three assertions hold:

1. The state $\mathrm{W}^{0}$ is $L^{\infty}$-controllable with respect to system (2.2), (2.3) at a given time $T>0$ iff $\mathrm{Z}^{0}$ is $L^{\infty}$-controllable with respect to system (4.1), (4.2) at the same time.

2. The state $\mathrm{W}^{0}$ is approximately $L^{\infty}$-controllable with respect to system (2.2), (2.3) at a given time $T>0$ iff $\mathrm{Z}^{0}$ is approximately $L^{\infty}$-controllable with respect to system (4.1), (4.2) at the same time.

3. The state $\mathrm{W}^{0}$ is approximately $L^{\infty}$-controllable with respect to system (2.2), (2.3) at a free time iff $\mathrm{Z}^{0}$ is approximately $L^{\infty}$-controllable with respect to system (4.1), (4.2) at a free time.

Thus, 2-d control system (2.2), (2.3) replicates the controllability properties of 1-d control system (4.1), (4.2) and vise versa.

\section{Auxiliary Control Problem}

In this section we study auxiliary control problem (4.1), (4.2). Using the Fourier transform method, by analogy with [4, Proposition 3.2 and Lemma 6.7], we obtain the following two propositions.

Proposition 5.1. Let $\mathrm{Z}^{0} \in \widehat{\mathbf{H}}^{1[1 / 2]}, u \in L^{\infty}(0, T), \mathcal{U}(t)=u(t)(H(t)-H(t-$ $T))-u(-t)(H(t+T)-H(t))$, and $\partial^{-1} \mathcal{U}(t)=\int_{-\infty}^{t} \mathcal{U}(\mu) d \mu, t \in \mathbb{R}$. Then,

$$
\mathrm{Z}(\cdot, T)=\mathcal{E}(\cdot, T) *\left(\mathrm{Z}^{0}-\left(\begin{array}{c}
\partial^{-1} \mathcal{U} \\
\operatorname{sgn} \xi \mathcal{U}
\end{array}\right)\right)
$$

where $\mathrm{Z}$ is the unique solution to (4.1), (4.2), * is the convolution with respect to $\xi$, and

$$
\begin{aligned}
\mathcal{E}(\cdot, T) & =\frac{1}{2}\left(\begin{array}{cc}
\delta(\xi+T)+\delta(\xi-T) & H(\xi+T)-H(\xi-T) \\
\delta^{\prime}(\xi+T)-\delta^{\prime}(\xi-T) & \delta(\xi+T)+\delta(\xi-T)
\end{array}\right) \\
& =\frac{1}{\sqrt{2 \pi}} \mathcal{F}^{-1}\left(\begin{array}{cc}
\cos (T \rho) & \frac{\sin (T \rho)}{\rho} \\
\rho \sin (T \rho) & \cos (T \rho)
\end{array}\right) .
\end{aligned}
$$

Proposition 5.2. Let $\mathcal{E}$ be defined by $(5.2), s \in \mathbb{R}$. Then we have

$$
\left\|\mathcal{E}(\cdot, T) * f\left|\left\|^{s[1 / 2]} \leq \sqrt{2 T^{2}+6}\right\|\right| f\right\|^{s[1 / 2]}, \quad f \in \widehat{\mathbf{H}}^{s[1 / 2]} .
$$


Theorem 5.3. A state $\mathrm{Z}^{0} \in \widehat{\mathbf{H}}^{1[1 / 2]}$ is approximately $L^{\infty}$-controllable with respect to system (4.1), (4.2) at a given time $T>0$ iff

$$
\begin{gathered}
\mathrm{Z}_{1}^{0}=\operatorname{sgn} \xi \mathrm{Z}_{0}^{0 \prime}, \\
\operatorname{supp} \mathrm{Z}^{0} \subset[-T, T] .
\end{gathered}
$$

Moreover, the controls $u_{n}(t)=\mathrm{Z}_{0}^{0 \prime}(t n /(n-1)) * n \varphi(t n), t \in[0, T], n=\overline{2, \infty}$, solve the approximate $L^{\infty}$-controllability problem with respect to system (4.1), (4.2) at a given time $T>0$ for the state $\mathrm{Z}^{0}$, where $\varphi \in C^{1}(\mathbb{R})$ is the function determined by (7.5).

P r o o f. Necessity of (5.5), (5.6). Let $\mathrm{Z}^{0}$ be approximately $L^{\infty}$-controllable with respect to system (4.1), (4.2) at a given time $T>0$. Then there exists a sequence of controls $\left\{u_{n}\right\}_{n=1}^{\infty} \subset L^{\infty}(0, T)$ such that $\left\|\mathrm{Z}^{n}(\cdot, T)\right\| \|^{1[1 / 2]} \rightarrow 0$ as $n \rightarrow \infty$, where $\mathrm{Z}^{n}$ is the unique solution to (4.1), (4.2) for $u=u_{n}, n=\overline{1, \infty}$. According to Propositions 5.1 and 5.2, we get

$$
\left|\mathrm{Z}_{0}^{0}-\partial^{-1} \mathcal{U}_{n}\right|^{1[1 / 2]} \rightarrow 0 \quad \text { and } \quad\left|\mathrm{Z}_{1}^{0}-\operatorname{sgn} \xi \mathcal{U}_{n}\right|^{0[1 / 2]} \rightarrow 0 \quad \text { as } n \rightarrow \infty,
$$

where $\mathcal{U}_{n}(t)=u_{n}(t)(H(t)-H(t-T))-u_{n}(-t)(H(t+T)-H(t))$, and $\partial^{-1} \mathcal{U}_{n}(t)=$ $\int_{-\infty}^{t} \mathcal{U}_{n}(\mu) d \mu, t \in \mathbb{R}, n=\overline{1, \infty}$. Taking into account Lemma 7.2, we get

$$
\left|\operatorname{sgn} \xi\left(\mathrm{Z}_{0}^{0 \prime}-\mathcal{U}_{n}\right)\right|^{0[1 / 2]} \rightarrow 0 \quad \text { as } n \rightarrow \infty .
$$

Comparing (5.7) and (5.8), it is seen that (5.5) holds. Note that supp $\partial^{-1} \mathcal{U}_{n} \subset$ $[-T, T], n=\overline{1, \infty}$. From (5.7) it follows that the sequence $\left\{\partial^{-1} \mathcal{U}_{n}\right\}_{n=1}^{\infty}$ converges weakly to $\mathrm{Z}_{0}^{0}$ in $H_{0}^{0[1 / 2]}$. Hence (5.6) also holds.

Sufficiency of (5.5), (5.6). Put $\widetilde{\mathcal{U}}_{n}(t)=\mathrm{Z}_{0}^{0}(n t / n-1) * n \varphi(n t), \mathcal{U}_{n}(t)=\widetilde{\mathcal{U}}_{n}^{\prime}(t)$, $t \in \mathbb{R}, n=\overline{2, \infty}$. Here $\varphi \in C^{1}(\mathbb{R})$ is the function determined by (7.5). Due to Lemma 7.4, we see that $\operatorname{supp} \mathcal{U}_{n} \subset[-T, T], n=\overline{2, \infty}$, and (5.7) holds. Applying Propositions 5.1 and 5.2, from here we get

$$
\|\left.\left|\mathrm{Z}^{n}(\cdot, T)\right|\right|^{1[1 / 2]} \rightarrow 0 \quad \text { as } n \rightarrow \infty,
$$

where $\mathrm{Z}^{n}$ is the unique solution to (4.1), (4.2) with $u(t)=u_{n}(t):=\mathcal{U}_{n}(t), t \in$ $[0, T], n=\overline{2, \infty}$. Thus, the controls $u_{n}, n=\overline{2, \infty}$, solve the approximate $L^{\infty}$ controllability problem for the state $\mathrm{Z}^{0}$. The theorem is proved.

Analyzing the proof of Theorem 5.3, we obtain

Corollary 5.4. A state $\mathrm{Z}^{0} \in \widehat{\mathbf{H}}^{1[1 / 2]}$ is $L^{\infty}$-controllable with respect to system (4.1), (4.2) at a given time $T>0$ iff (5.5), (5.6) hold and

$$
\mathrm{Z}_{1}^{0} \in L^{\infty}(\mathbb{R})
$$


Moreover, under conditions (5.5), (5.6), and (5.9) the control $u(t)=\mathrm{Z}_{0}^{0 \prime}(t), t \in$ $[0, T]$, solves the approximate $L^{\infty}$-controllability problem with respect to system (4.1), (4.2) at the time $T>0$ for $\mathrm{Z}^{0}$.

Theorem 5.5. A state $\mathrm{Z}^{0} \in \widehat{\mathbf{H}}^{1[1 / 2]}$ is approximately $L^{\infty}$-controllable with respect to system (4.1), (4.2) at a free time iff (5.5) holds.

P r o o f. Necessity of (5.5). Let $\mathrm{Z}^{0}$ be approximately $L^{\infty}$-controllable with respect to system (4.1), (4.2) at a free time. Then there exist a sequence $\left\{T_{n}\right\}_{n=1}^{\infty} \subset(0,+\infty)$ and a sequence $\left\{u_{n}\right\}_{n=1}^{\infty}, u_{n} \in L^{\infty}\left(0, T_{n}\right), n=\overline{1, \infty}$, such that

$$
\left\|\mathrm{Z}^{n}\left(\cdot, T_{n}\right)\right\|^{1[1 / 2]} \rightarrow 0 \quad \text { as } n \rightarrow \infty,
$$

where $\mathrm{Z}^{n}=\left(\begin{array}{l}\mathrm{Z}_{0}^{n} \\ \mathrm{Z}_{1}^{n}\end{array}\right)$ is the unique solution to (4.1), (4.2) with $T=T_{n}$ and $u=u_{n}$, $n=\overline{1, \infty}$. Put $\mathcal{U}_{n}(t)=u_{n}(t)\left(H(t)-H\left(t-T_{n}\right)\right)-u_{n}(-t)\left(H\left(t+T_{n}\right)-H(t)\right)$, $t \in \mathbb{R}, n=\overline{1, \infty}$. According to Proposition 5.1, we have

$$
\begin{aligned}
2\left(\mathrm{Z}_{0}^{n}\left(x, T_{n}\right)\right)_{x}= & \left(\mathrm{Z}_{0}^{0 \prime}-\mathcal{U}_{n}\right)\left(x+T_{n}\right)+\left(\mathrm{Z}_{0}^{0 \prime}-\mathcal{U}_{n}\right)\left(x-T_{n}\right) \\
& +\left(\mathrm{Z}_{1}^{0}-\operatorname{sgn} \xi \mathcal{U}_{n}\right)\left(x+T_{n}\right)-\left(\mathrm{Z}_{1}^{0}-\operatorname{sgn} \xi \mathcal{U}_{n}\right)\left(x-T_{n}\right), \\
2 \mathrm{Z}_{1}^{n}\left(x, T_{n}\right)= & \left(\mathrm{Z}_{0}^{0 \prime}-\mathcal{U}_{n}\right)\left(x+T_{n}\right)-\left(\mathrm{Z}_{0}^{0 \prime}-\mathcal{U}_{n}\right)\left(x-T_{n}\right) \\
& +\left(\mathrm{Z}_{1}^{0}-\operatorname{sgn} \xi \mathcal{U}_{n}\right)\left(x+T_{n}\right)+\left(\mathrm{Z}_{1}^{0}-\operatorname{sgn} \xi \mathcal{U}_{n}\right)\left(x-T_{n}\right) .
\end{aligned}
$$

Therefore,

$$
\begin{aligned}
2\left(\mathrm{Z}_{0}^{0 \prime}-\mathcal{U}_{n}\right)(x)= & \left(\mathrm{Z}_{0}^{n}\left(x-T_{n}, T_{n}\right)+\mathrm{Z}_{0}^{n}\left(x+T_{n}, T_{n}\right)\right)_{x} \\
& +\mathrm{Z}_{1}^{n}\left(x-T_{n}, T_{n}\right)-\mathrm{Z}_{1}^{n}\left(x+T_{n}, T_{n}\right) \\
2\left(\mathrm{Z}_{1}^{0}-\operatorname{sgn} \xi \mathcal{U}_{n}\right)(x)= & \left(\mathrm{Z}_{0}^{n}\left(x-T_{n}, T_{n}\right)-\mathrm{Z}_{0}^{n}\left(x+T_{n}, T_{n}\right)\right)_{x} \\
& +\mathrm{Z}_{1}^{n}\left(x-T_{n}, T_{n}\right)+\mathrm{Z}_{1}^{n}\left(x+T_{n}, T_{n}\right) .
\end{aligned}
$$

Taking into account (5.10), we obtain

$$
\left|\mathrm{Z}_{0}^{0 \prime}-\partial^{-1} \mathcal{U}_{n}\right|^{0[1 / 2]} \rightarrow 0 \quad \text { and } \quad\left|\mathrm{Z}_{1}^{0}-\operatorname{sgn} \xi \mathcal{U}_{n}\right|^{0[1 / 2]} \rightarrow 0 \quad \text { as } n \rightarrow \infty
$$

Applying Lemma 7.2, we obtain (5.5).

Sufficiency of (5.5). Let $\left\{T_{n}\right\}_{n=1}^{\infty} \subset(0,+\infty)$ be a nondecreasing sequence under the condition $T_{n} \rightarrow \infty$ as $n \rightarrow \infty$. Let $\psi \in C^{2}(\mathbb{R})$ be an even function such that $0 \leq \psi(\xi) \leq 1, \xi \in \mathbb{R} ; \psi(\xi)=1,|\xi| \leq 1 / 2 ; \psi(\xi)=0,|\xi| \geq 1$. Put $\widehat{\mathrm{Z}}_{0}^{n}(\xi)=\mathrm{Z}_{0}^{0}(\xi) \psi\left(\xi / T_{n}\right), \widehat{\mathrm{Z}}_{1}^{n}=\operatorname{sgn} \xi \widehat{\mathrm{Z}}_{0}^{n \prime}, \xi \in \mathbb{R}^{n}, n=\overline{1, \infty}$. Due to Lemmas 7.2 and 7.5, we obtain $\widehat{Z}^{n}=\left(\begin{array}{l}\widehat{Z}_{0}^{n} \\ \widehat{Z}_{1}^{n}\end{array}\right) \in \widehat{\mathbf{H}}^{1[1 / 2]}, n=\overline{1, \infty}$. Evidently, condition (5.5) 
holds for $\widehat{\mathrm{Z}}^{n}, n=\overline{1, \infty}$. For each $n=\overline{1, \infty}$, applying Theorem 5.3 , we can find some controls $u_{k}^{n} \in L^{\infty}\left(0, T_{n}\right), k=\overline{2, \infty}$, such that $\left\|\mathrm{Z}_{k}^{n}\right\|^{1[1 / 2]} \rightarrow 0$ as $n \rightarrow \infty$, where $\mathrm{Z}_{k}^{n}$ is the unique solution to (4.1), (4.2) with $T=T_{n}, u=u_{k}^{n}$, and $\mathrm{Z}^{0}=\mathrm{Z}^{n}$, $k=\overline{2, \infty}$. For each $n=\overline{1, \infty}$, set $k_{n}=\overline{2, \infty}$ such that

$$
\left\|\mathrm{Z}_{k_{n}}^{n}\right\|^{1[1 / 2]} \leq \frac{1}{n}
$$

and denote by $\mathrm{Z}^{n}$ the unique solution to control problem (4.1), (4.2) with $T=T_{n}$, $u=u_{k_{n}}^{n}$, and the given initial state $\mathrm{Z}^{0}$. Then $\mathrm{Z}^{n}-\widehat{\mathrm{Z}}^{n}$ is the unique solution to (4.1), (4.2) with $T=T_{n}, u=u_{k_{n}}^{n}$, and the initial state $\mathrm{Z}^{0}-\widehat{\mathrm{Z}}^{n}, n=\overline{1, \infty}$. For $n=\overline{1, \infty}$, taking into account Proposition 5.1, we get

$$
\begin{aligned}
\mathrm{Z}^{n}\left(\xi, T_{n}\right) & -\mathrm{Z}_{k_{n}}^{n}\left(\xi, T_{n}\right) \\
& =\left(\begin{array}{c}
H\left(\xi+T_{n}\right)\left(\mathrm{Z}_{0}^{0}-\widehat{\mathrm{Z}}_{0}^{n}\right)\left(\xi+T_{n}\right)+H\left(T_{n}-\xi\right)\left(\mathrm{Z}_{0}^{0}-\widehat{\mathrm{Z}}_{0}^{n}\right)\left(T_{n}-\xi\right) \\
H\left(\xi+T_{n}\right)\left(\mathrm{Z}_{0}^{0}-\widehat{\mathrm{Z}}_{0}^{n}\right)^{\prime}\left(\xi+T_{n}\right)+H\left(T_{n}-\xi\right)\left(\mathrm{Z}_{0}^{0}-\widehat{\mathrm{Z}}_{0}^{n}\right)^{\prime}\left(T_{n}-\xi\right)
\end{array}\right) .
\end{aligned}
$$

According to Lemmas 7.2 and 7.7, we obtain

$$
\left\|\mid \mathrm{Z}^{n}-\mathrm{Z}_{k_{n}}^{n}\right\|^{1[1 / 2]} \rightarrow 0 \quad \text { as } n \rightarrow \infty .
$$

Summarizing (5.12) and (5.13), we see that

$$
\left\|\left.\left|\mathrm{Z}^{n}\right|\right|^{1[1 / 2]} \leq\right\|\left|\mathrm{Z}^{n}-\mathrm{Z}_{k_{n}}^{n}\right|\left\|^{1[1 / 2]}+\right\| \mid \mathrm{Z}_{k_{n}}^{n} \|^{\left.1\right|^{1[1 / 2]} \rightarrow \infty \quad \text { as } n \rightarrow \infty .}
$$

Thus, the controls $u_{k_{n}}^{n}, n=\overline{1, \infty}$, solve the approximate $L^{\infty}$-controllability problem with respect to system (4.1), (4.2) at a free time for $\mathrm{Z}^{0}$. The theorem is proved.

\section{Main Control Problem}

In this section we study control system (2.2), (2.3) using the results of Secs. 4 and 5. Theorems 3.3, 4.1, 5.3, 5.5 and Corollaries 4.2, 4.5, 5.4 yield the following three theorems.

Theorem 6.1. A state $\mathrm{W}^{0} \in \widehat{\mathbf{H}}^{1}$ is $L^{\infty}$-controllable with respect to system (2.2), (2.3) at a given time $T>0$ iff

$$
\begin{gathered}
\mathrm{W}^{0} \in \widehat{\mathbb{H}}^{1}, \\
\mathrm{~W}_{1}^{0}=\boldsymbol{\Phi}\left(\operatorname{sgn} x \frac{d}{d \xi}\left(\boldsymbol{\Phi}^{-1} \mathrm{~W}_{0}^{0}\right)\right), \\
\operatorname{supp} \mathrm{W}^{0} \in D_{T}, \\
\boldsymbol{\Phi}^{-1} \mathrm{~W}_{1}^{0} \subset L^{\infty}(\mathbb{R}),
\end{gathered}
$$

where $D_{T}=\left\{x \in \mathbb{R}^{2}|| x \mid \leq T\right\}$. 
Theorem 6.2. A state $\mathrm{W}^{0} \in \widehat{\mathbf{H}}^{1}$ is approximately $L^{\infty}$-controllable with respect to system (2.2), (2.3) at a given time $T>0$ iff (6.1)-(6.3) hold.

Theorem 6.3. A state $\mathrm{W}^{0} \in \widehat{\mathbf{H}}^{1}$ is approximately $L^{\infty}$-controllable with respect to system (2.2), (2.3) at a free time iff (6.1) and (6.2) hold.

$\mathrm{E} \mathrm{x}$ a m p l e 6.1. Let $\mathrm{w}_{0}^{0}(r)=\frac{8}{3} H\left(1-r^{2}\right)\left(1-r^{2}\right)^{3 / 2}, \mathrm{w}_{1}^{0}(r)=2 H(1-$ $\left.r^{2}\right)\left(\left(2-3 r^{2}\right) \ln \frac{1+\sqrt{1-r^{2}}}{r}-3 \sqrt{1-r^{2}}\right), r \in \mathbb{R}$, and $\mathrm{W}_{j}^{0}(x)=\mathrm{w}_{j}^{0}(|x|), x \in \mathbb{R}^{2}$, $j=0,1$. Evidently, conditions (6.1) and (6.3) hold for $\mathrm{W}=\left(\begin{array}{c}\mathrm{W}_{0}^{0} \\ \mathrm{~W}_{1}^{0}\end{array}\right)$ and $T>1$. According to Theorem 3.5, replacing $\sqrt{r^{2}-\xi^{2}}$ by $p$, we get

$$
\begin{aligned}
& \left(\boldsymbol{\Phi}^{-1} \mathrm{~W}_{0}^{0}\right)(\xi)=\frac{8}{3} \sqrt{\frac{2}{\pi}} H\left(1-\xi^{2}\right) \int_{\xi}^{1} \frac{r\left(1-r^{2}\right)^{3 / 2}}{\sqrt{r^{2}-\xi^{2}}} d r \\
& \quad=\frac{8}{3} \sqrt{\frac{2}{\pi}} H\left(1-\xi^{2}\right) \int_{0}^{\sqrt{1-\xi^{2}}}\left(1-\xi^{2}-p^{2}\right) d p=\sqrt{\frac{2}{\pi}}\left(1-\xi^{2}\right)^{2}, \quad \xi \in \mathbb{R} .
\end{aligned}
$$

Therefore, (6.4) also holds. Let us verify (6.2). Taking into account Theorem 3.4, replacing $\xi$ by $r \cosh p$, we obtain

$$
\begin{gathered}
\mathbb{I}_{0}^{0} \boldsymbol{\Phi}\left(\operatorname{sgn} \xi \frac{d}{d \xi}\left(\Phi^{-1} \mathrm{~W}_{0}^{0}\right)\right)=H\left(1-r^{2}\right) \int_{r}^{1} \frac{4-12 \xi}{\sqrt{\xi^{2}-t^{2}}} \\
=H\left(1-r^{2}\right) \int_{0}^{\ln \frac{1+\sqrt{1-r^{2}}}{|r|}}\left(4-12 r^{2} \cosh ^{2} p\right) d p \\
=2 H\left(1-r^{2}\right)\left(\left(2-3 r^{2}\right) \ln \frac{1+\sqrt{1-r^{2}}}{r}-3 \sqrt{1-r^{2}}\right)=\mathrm{w}_{1}^{0}(r), \quad r \in \mathbb{R} .
\end{gathered}
$$

Hence (6.2) is also valid. Thus, the state $\mathrm{W}^{0}$ is (approximately) $L^{\infty}$-controllable with respect to system $(2.2),(2.3)$ at a given time $T>1$ according to Theorems 6.1 and 6.2. Since $\left(\boldsymbol{\Phi}^{-1} \mathrm{~W}_{0}^{0}\right)^{\prime} \in L^{\infty}(\mathbb{R})$ (see (6.5)), we conclude that $u(t)=$ $\left(\boldsymbol{\Phi}^{-1} \mathrm{~W}_{0}^{0}\right)^{\prime}(t)=-4 \sqrt{\frac{\pi}{2}} H\left(1-t^{2}\right) t\left(1-t^{2}\right), t \in[0, T]$, solves the (approximate) $L^{\infty}$-controllability problem with respect to system $(2.2),(2.3)$ at the time $T>1$ for $\mathrm{W}_{0}^{0}$.

$\mathrm{Ex} \mathrm{a} \mathrm{m} \mathrm{p} \mathrm{l} \mathrm{e} \mathrm{6.2.} \mathrm{Let} \mathrm{w}_{0}^{0}(r)=\frac{1}{\left(1+r^{2}\right)^{3 / 2}}, \mathrm{w}_{1}^{0}(r)=\frac{2}{\pi}\left(\frac{r^{2}-2}{\left(1+r^{2}\right)^{5 / 2}} \ln \frac{\sqrt{1+r^{2}}+1}{\sqrt{1+r^{2}}-1}+\right.$ $\left.\frac{3}{2\left(1+r^{2}\right)^{2}}\right), r \in \mathbb{R}$, and $\mathrm{W}_{j}^{0}(x)=\mathrm{w}_{j}^{0}(|x|), x \in \mathbb{R}^{2}, j=0,1$. Evidently, condition (6.1) holds for $\mathrm{W}=\left(\begin{array}{l}\mathrm{W}_{0}^{0} \\ \mathrm{~W}_{1}^{0}\end{array}\right)$. Let us verify (6.2). According to Theorem 3.5, 
replacing $\sqrt{r^{2}-\xi^{2}}$ by $p$, we get

$$
\begin{aligned}
\left(\boldsymbol{\Phi}^{-1} \mathrm{~W}_{0}^{0}\right)(\xi)=\sqrt{\frac{2}{\pi}} \int_{\xi}^{\infty} \frac{r d r}{\left(1+r^{2}\right)^{3 / 2} \sqrt{r^{2}-\xi^{2}}} & \\
& =\sqrt{\frac{2}{\pi}} \int_{0}^{\infty} \frac{d p}{\left(1+\xi^{2}+p^{2}\right)^{3 / 2}}=\sqrt{\frac{2}{\pi}} \frac{1}{1+\xi^{2}}, \quad \xi \in \mathbb{R} .
\end{aligned}
$$

According to Theorem 6.2 and (6.6), the state $\mathrm{W}^{0}$ is not approximately $L^{\infty}$ controllable with respect to system $(2.2),(2.3)$ at any given time $T>0$. Taking into account Theorem 3.4, by replacing $\xi$ by $r \cosh v$ and substituting $\tanh v=p$ into (6.6), we obtain

$$
\begin{gathered}
\frac{\pi}{2} \mathbb{I}_{0}^{0} \boldsymbol{\Phi}\left(\operatorname{sgn} \xi \frac{d}{d \xi}\left(\Phi^{-1} \mathrm{~W}_{0}^{0}\right)\right)=\int_{r}^{\infty} \frac{\left(3 \xi^{2}-1\right) d \xi}{\left(1+\xi^{2}\right)^{3} \sqrt{\xi^{2}-r^{2}}} \\
=\int_{0}^{\infty} \frac{3 r^{2} \cosh ^{2} v-1}{\left(1+r^{2} \cosh ^{2} v\right)^{3}} d v=\int_{0}^{1} \frac{\left(3 r^{2}-1+p^{2}\right)\left(1-p^{2}\right)}{\left(1+r^{2}-p^{2}\right)^{3}} \\
=-4 r^{4} \int_{0}^{1} \frac{d p}{\left(1+r^{2}-p^{2}\right)^{3}}+5 r^{2} \int_{0}^{1} \frac{d p}{\left(1+r^{2}-p^{2}\right)^{2}}-\int_{0}^{1} \frac{d p}{1+r^{2}-p^{2}} \\
=\frac{3}{2\left(1+r^{2}\right)^{2}}+\frac{r^{2}-2}{\left(1+r^{2}\right)^{5 / 2}} \ln \frac{\sqrt{1+r^{2}}+1}{\sqrt{1+r^{2}}-1}=\frac{\pi}{2} \mathrm{w}_{1}^{0}(r), \quad r \in \mathbb{R} .
\end{gathered}
$$

Hence (6.2) holds. Thus, the state $\mathrm{W}^{0}$ is approximately $L^{\infty}$-controllable with respect to system $(2.2),(2.3)$ at a free time according to Theorems 6.3. Now, let us construct some controls solving the approximate $L^{\infty}$-controllability problem with respect to system $(2.2),(2.3)$ at a free time. Let $\psi \in C^{2}(\mathbb{R})$ be an even function such that $\psi(\xi)=1$ if $|\xi| \leq 1 / 2, \psi(\xi)=0$ if $|\xi|>1$, and $0 \leq|\xi| \leq 1$ if $1 / 2 \leq$ $|\xi| \leq 1$. With regard to $(6.6)$, we get $\left(\boldsymbol{\Phi}^{-1} \mathrm{~W}_{0}^{0}\right)^{\prime} \in L^{\infty}(\mathbb{R})$. According to Lemma 7.7, we see that the controls $u(t)=\left(\boldsymbol{\Phi}^{-1} \mathrm{~W}_{0}^{0}\right)^{\prime}(t) \psi\left(t / T_{n}\right)=-\sqrt{\frac{2}{\pi}} \frac{2 t}{\left(1+t^{2}\right)^{2}} \psi\left(t / T_{n}\right)$, $t \in\left[0, T_{n}\right], n=\overline{1, \infty}$, solve the problem mentioned above for each sequence $\left\{T_{n}\right\}_{n=1}^{\infty} \subset(0,+\infty)$ such that $T_{n} \rightarrow \infty$ as $n \rightarrow \infty$.

\section{Auxiliary Statements}

In this section we prove some auxiliary assertions used in Secs. 2-6.

Lemma 7.1. If $\widehat{F} \in H_{s}^{0}$ and $F=\widehat{F} / \sqrt{|\rho|}$, then $F \in H_{1 / 2+s}^{-3 / 2}, s \in \mathbb{R}$.

P r o o f. Evidently, $\rho F \in H_{s-1 / 2}^{0}$. Set $F_{0}=\left(1+\rho^{2}\right)^{s / 2-1 / 4} F, f_{0}=\mathcal{F}^{-1} F_{0}$. Then, $\rho F_{0} \in H_{0}^{0}$ and $f_{0}^{\prime} \in H_{0}^{0}$. Put $g(x)=f_{0}^{\prime}(x)$ and $\partial^{-1} g(x)=\int_{0}^{x} g(\xi) d \xi$, 
$x \in \mathbb{R}$. We have

$$
\left|\partial^{-1} g(x)\right| \leq\|g\|_{0}^{0} \sqrt{\int_{0}^{|x|} d \xi}=\sqrt{|x|}\|g\|_{0}^{0}, \quad x \in \mathbb{R} .
$$

Therefore,

$$
\left\|\partial^{-1} g\right\|_{-3 / 2}^{0} \leq\|g\|_{0}^{0} \sqrt{2 \int_{0}^{|x|} \frac{x d x}{\left(1+x^{2}\right)^{3 / 2}}}=\sqrt{2}\|g\|_{0}^{0} .
$$

Hence,

$$
\left\|\partial^{-1} g\right\|_{-3 / 2}^{1} \leq\left(\left(\left\|\partial^{-1} g\right\|_{-3 / 2}^{0}\right)+\left(\|g\|_{0}^{0}\right)^{2}\right)^{1 / 2} \leq \sqrt{3}\|g\|_{0}^{0} .
$$

Since there exists $C_{0} \in \mathbb{C}$ such that $f_{0}=\partial^{-1} g+C_{0}$, it is seen that $f_{0} \in H_{-3 / 2}^{1}$, $F_{0} \in H_{1}^{-3 / 2}$. Hence, $F=\left(1+\rho^{2}\right)^{1 / 4-s / 2} \in H_{1 / 2}^{-3 / 2}$. The lemma is proved.

Lemma 7.2. If $f \in H_{0}^{0[1 / 2]}$ is odd, then $\operatorname{sgn} x f \in H_{0}^{0[1 / 2]}$ and $|\operatorname{sgn} x f|_{0}^{0[1 / 2]} \leq$ $\sqrt{2}|f|_{0}^{0[1 / 2]}$.

P r o o f. Set $g=\operatorname{sgn} x f$. Taking into account (3.3), we obtain

$$
\begin{aligned}
\left(|f|_{0}^{0[1 / 2]}\right)^{2} & =\int_{-\infty}^{\infty} \int_{-\infty}^{\infty} \frac{|f(x)-f(y)|^{2}}{|x-y|^{2}} d y d x \\
& =2 \int_{0}^{\infty} \int_{0}^{\infty} \frac{|f(x)-f(y)|^{2}}{|x-y|^{2}} d y d x+2 \int_{0}^{\infty} \int_{0}^{\infty} \frac{|f(x)+f(y)|^{2}}{|x+y|^{2}} d y d x
\end{aligned}
$$

Since $|x+y| \geq|x-y|, x \geq 0, y \geq 0$, we see that

$$
\begin{aligned}
\left(|g|_{0}^{0[1 / 2]}\right)^{2} & =\int_{-\infty}^{\infty} \int_{-\infty}^{\infty} \frac{|g(x)-g(y)|^{2}}{|x-y|^{2}} d y d x \leq 4 \int_{0}^{\infty} \int_{0}^{\infty} \frac{|g(x)-g(y)|^{2}}{|x-y|^{2}} d y d x \\
& =4 \int_{0}^{\infty} \int_{0}^{\infty} \frac{|f(x)-f(y)|^{2}}{|x-y|^{2}} d y d x \leq 2\left(|f|_{0}^{0[1 / 2]}\right)^{2} .
\end{aligned}
$$

That was to be proved.

Lemma 7.3. Let $f \in H_{0}^{1[1 / 2]}$ and $\operatorname{supp} f \subset[-\alpha, \alpha]$. Let $f_{n}(x)=f\left(\frac{n x}{n-1}\right)$, $x \in \mathbb{R}, n=\overline{2, \infty}$. Then, $\operatorname{supp} f_{n} \subset[-\alpha+1 / n, \alpha-1 / n],\left[f_{n}\right] \in H_{0}^{1 /[1 / 2]}, n=\overline{2, \infty}$, and

$$
\left|f-f_{n}\right|_{0}^{1[1 / 2]} \rightarrow 0 \quad \text { as } n \rightarrow \infty .
$$


P r o o f. Evidently, $\operatorname{supp} f_{n} \subset[-\alpha+1 / n, \alpha-1 / n], n=\overline{2, \infty}$. Put $F=\mathcal{F} f$ and $F_{n}=\mathcal{F} f_{n}, n=\overline{2, \infty}$. Then, $F_{n}(\rho)=\frac{n-1}{n} F_{0}\left(\frac{n-1}{n} \rho\right), \rho \in \mathbb{R}, n=\overline{2, \infty}$. Therefore, for $n=\overline{2, \infty}$, we have

$$
\begin{aligned}
\left|f_{n}\right|_{0}^{1[1 / 2]} & =\left|F_{n}\right|_{s[1 / 2]}^{0}=\frac{n-1}{n}\left(\int_{-\infty}^{\infty}\left(1+\rho^{2}\right)|\rho|\left|F\left(\frac{n-1}{n} \rho\right)\right|^{2} d \rho\right)^{1 / 2} \\
& \leq \frac{n}{n-1}\left(\int_{-\infty}^{\infty}\left(1+\xi^{2}\right)|\xi||F(\xi)|^{2} d \xi\right)^{1 / 2}=\frac{n}{n-1}|f|_{0}^{1[1 / 2]}
\end{aligned}
$$

Thus, $f_{n} \in H_{0}^{1 /[1 / 2]}, n=\overline{2, \infty}$. Now, let us prove assertion (7.1). We have

$$
\left|f-f_{n}\right|_{0}^{1[1 / 2]} \leq\left|F-F_{n}\right|_{1[1 / 2]}^{0}+\frac{1}{n-1}\left|F_{n}\right|_{1[1 / 2]}^{0}, \quad n=\overline{2, \infty} .
$$

According to (7.2), the second summand in the right-hand side of this estimate tends to 0 as $n \rightarrow \infty$. Therefore, to prove (7.1) it is sufficient to prove

$$
\left|F-F_{n}\right|_{1[1 / 2]}^{0} \rightarrow 0 \quad \text { as } n \rightarrow \infty \text {. }
$$

We have

$$
\begin{aligned}
\left(\left|F-F_{n}\right|_{1[1 / 2]}^{0}\right)^{2} \leq & \int_{|\rho| \leq \sqrt[4]{n}}\left(1+\rho^{2}\right)|\rho|\left|F(\rho)-F_{0}\left(\frac{n-1}{n} \rho\right)\right|^{2} d \rho \\
& +\left|\left[H\left(\rho^{2}-\sqrt{n}\right)\left(F-\frac{n}{n-1} F_{n}\right)\right]\right|_{1[1 / 2]}^{0} .
\end{aligned}
$$

Taking into account Lemma 7.1, we see that $f \in H_{-3 / 2}^{3 / 2}$. Moreover, $f \in H_{0}^{0}$, because supp $f_{0} \subset[-\alpha, \alpha]$ and $H_{-3 / 2}^{3 / 2} \subset H_{-3 / 2}^{0}[10$, Chap. 1]. Then,

$$
\left|F^{\prime}(\rho)\right| \leq \int_{-\alpha}^{\alpha}|x f(x)| d x \leq \sqrt{\frac{3 \alpha^{3}}{2}}\|f\|_{0}^{0}, \quad \rho \in \mathbb{R} .
$$

Hence,

$$
\begin{aligned}
\int_{|\rho| \leq \sqrt[4]{n}} & \left(1+\rho^{2}\right)^{s}|\rho|\left|F_{0}(\rho)-F\left(\frac{n-1}{n} \rho\right)\right|^{2} d \rho \\
\leq & \frac{2}{n^{2}} \sup _{\xi \in \mathbb{R}}\left|f^{\prime}(\xi)\right|^{2} \int_{0}^{\sqrt[4]{n}}\left(\rho^{2}+\rho^{4}\right) \leq \frac{\alpha^{3}}{n^{3 / 4}}\left(\|f\|_{0}^{0}\right)^{2} \rightarrow 0 \quad \text { as } n \rightarrow \infty .
\end{aligned}
$$

Thus, the first summand in the right-hand side of (7.4) tends to 0 as $n \rightarrow \infty$. Since $F_{0} \in H_{1[1 / 2]}^{0}$ and $F_{n} \in H_{1[1 / 2]}^{0}, n=\overline{2, \infty}$, we see that the second summand also tends to 0 as $n \rightarrow \infty$ there. Hence (7.3) holds and (7.1) is valid. That was to be proved. 
Lemma 7.4. Let $f \in H_{0}^{1[1 / 2]}$ and $\operatorname{supp} f \subset[-\alpha, \alpha]$. Let $f_{n}=g_{n} * \varphi_{n}$, where $g_{n}(x)=f\left(\frac{n x}{n-1}\right), \varphi_{n}(x)=n \varphi(n x), x \in \mathbb{R}$,

$$
\varphi(x)=2\left[\begin{array}{ll}
0 & \text { if }|x| \geq 1 \\
(|x|-1)^{2} & \text { if } 1 / 2 \leq|x| \leq 1, \quad x \in \mathbb{R}, n=\overline{2, \infty} . \\
1 / 2-x^{2} & \text { if }|x| \leq 1 / 2
\end{array}\right.
$$

Then $\operatorname{supp} f_{n} \subset[-\alpha, \alpha],\left[f_{n}\right] \in H_{0}^{1 /[1 / 2]} \cap C^{1}(\mathbb{R}), n=\overline{2, \infty}$, and

$$
\left|f-f_{n}\right|_{0}^{1[1 / 2]} \rightarrow 0 \quad \text { as } n \rightarrow \infty .
$$

P r o o f. According to Lemma 7.3, we have supp $g_{n} \subset[-\alpha+1 / n, \alpha-1 / n]$, $g_{n} \in H_{0}^{1[1 / 2]}, n=\overline{2, \infty}$, and (7.1) holds. Since $\varphi_{n} \in C^{1}(\mathbb{R})$ and $\operatorname{supp} \varphi_{n} \subset$ $[-1 / n, 1 / n], n=\overline{2, \infty}$, we see that $\operatorname{supp} f_{n} \subset[-\alpha, \alpha], f_{n} \in H_{0}^{1 /[1 / 2]} \cap C^{1}(\mathbb{R})$, $n=\overline{2, \infty}$. Let us prove (7.6). Put $\Phi=\mathcal{F} \varphi$. We have

$$
\begin{aligned}
\Phi(\xi) & =\frac{4}{\sqrt{2 \pi}}\left(\int_{0}^{1 / 2}\left(1 / 2-x^{2}\right) \cos (\xi x) d x+\int_{1 / 2}^{1}(x-1)^{2} \cos (\xi x) d x\right) \\
& =\frac{8}{\sqrt{2 \pi}} \frac{2 \sin (\xi / 2)-\sin \xi}{\xi^{3}}=\frac{16}{\sqrt{2 \pi}} \frac{\sin (\xi / 2) \sin (\xi / 4)}{\xi^{3}}, \quad \xi \in \mathbb{R} .
\end{aligned}
$$

Using the Tailor formula, we get

$$
|\sqrt{2 \pi} \Phi(\xi)-1|=\left|8 \frac{2 \sin (\xi / 2)-\sin \xi}{\xi^{3}}-1\right| \leq \frac{|\xi|}{2}, \quad \xi \in \mathbb{R} .
$$

Therefore,

$$
|\sqrt{2 \pi} \Phi(\xi)-1|=\left|16 \frac{\sin (\xi / 2) \sin (\xi / 4)}{\xi^{3}}-1\right| \leq \frac{3}{2}, \quad \xi \in \mathbb{R} .
$$

We have

$$
\left|f-f_{n}\right|_{0}^{1[1 / 2]} \leq\left|f-g_{n}\right|_{0}^{1[1 / 2]}+\left|g_{n}-f_{n}\right|_{0}^{1[1 / 2]}, \quad n=\overline{2, \infty} .
$$

Put $F=\mathcal{F} f, G_{n}=\mathcal{F} g_{n}, \Phi_{n}=\mathcal{F} \varphi_{n}, n=\overline{2, \infty}$. Then $\Phi_{n}(\rho)=\Phi\left(\frac{\rho}{n}\right), G_{n}(\rho)=$ $\frac{n-1}{n} F_{0}\left(\frac{n-1}{n} \rho\right), n=\overline{2, \infty}$. Taking into account (7.2), applying (7.7) for $|\rho| \leq \sqrt{n}$ and (7.8) for $|\rho| \geq \sqrt{n}$, we get

$$
\left|f-g_{n}\right|_{0}^{1[1 / 2]}=\left|F_{n}\left(1-\sqrt{2 \pi} \Phi_{n}\right)\right|_{1[1 / 2]}^{0}
$$




$$
\begin{aligned}
\leq & \frac{1}{2}\left(\int_{|\rho| \leq \sqrt{n}}\left(1+\rho^{2}\right)^{s}|\rho|\left|F_{n}(\rho)\right|^{2} \frac{\rho^{2}}{n^{2}} d \rho\right. \\
& \left.+9 \int_{|\rho| \geq \sqrt{n}}\left(1+\rho^{2}\right)^{s}|\rho|\left|F_{n}(\rho)\right|^{2} \frac{\rho^{2}}{n^{2}} d \rho\right)^{1 / 2} \\
\leq & \frac{1}{2 \sqrt{n-1}}\left(|F|_{1[1 / 2]}^{0}+3 \sqrt{n}\left|H\left(\xi^{2}-(n-1)^{2} / n\right) F\right|_{1[1 / 2]}^{0}\right) .
\end{aligned}
$$

Since $F \in H_{1[1 / 2]}^{0}$, we have $\left|f-g_{n}\right|_{0}^{s[1 / 2]} \rightarrow 0$ as $n \rightarrow \infty$. Due to Lemma 7.3 , $\left|g_{n}-f_{n}\right|_{0}^{1[1 / 2]} \rightarrow 0$ as $n \rightarrow \infty$ too. With regard to (7.9), we obtain (7.6). That was to be proved.

Lemma 7.5. Let $\psi \in C^{1}(\mathbb{R})$ and $\operatorname{supp} \psi \in[-1,1]$. If $f \in H_{0}^{0[1 / 2]}$ and $\hat{f}_{a}(x)=$ $f(x) \psi(x / a), x \in \mathbb{R}, a>0$, then $\hat{f}_{a} \in H_{0}^{s[1 / 2]}, a>0$.

P r o o f. Taking into account (3.3), we have

$$
\left(|f|_{0}^{0[1 / 2]}\right)^{2}=\iint_{\mathbb{R}^{2}} \frac{|f(x)-f(y)|^{2}}{|x-y|^{2}} d y d x<\infty .
$$

Setting $I_{-\infty}=(-\infty,-a], I_{0}=[-a, a], I_{+\infty}=[a,+\infty$, we get

$$
\left(\left|\hat{f}_{a}\right|_{0}^{0[1 / 2]}\right)^{2}=\sum_{k, l=-\infty, 0,+\infty} J_{k, l}, \quad \text { where } J_{k, l}=\iint_{I_{k} \times I_{l}} \frac{\left|\hat{f}_{a}(x)-\hat{f}_{a}(y)\right|^{2}}{|x-y|^{2}} d y d x
$$

According to Lemma 7.1, $f \in L_{\text {loc }}^{2}(\mathbb{R})$. Set $M=\max \left\{\left|\psi^{\prime}(\xi)\right| \mid \xi \in[-a, a]\right\}$. From the mean value theorem it follows that $|\psi(x / a)-\psi(y / a)| \leq \frac{M}{a}(x-y)$, $(x, y) \in[-a, a]^{2}$. Then

$$
\begin{aligned}
J_{-\infty,-\infty}=J_{+\infty,+\infty} & =0 \\
J_{0,0} \leq 2 \iint_{I_{0} \times I_{0}} & \frac{|f(x)-f(y)|^{2}}{|x-y|^{2}} d y d x \\
& +2 \iint_{I_{0} \times I_{0}}|f(x)|^{2} \frac{|\psi(x / a)-\psi(y / a)|^{2}}{|x-y|^{2}} d y d x \\
\leq & 2\left(|f|_{0}^{0[1 / 2]}\right)^{2}+4 M\left(\|f\|_{L^{2}\left(I_{0}\right)}\right)^{2} \\
J_{-\infty, 0}=J_{0,-\infty}= & \iint_{I_{0} \times I_{-\infty}} \frac{|f(x) \psi(x / a)|^{2}}{|x-y|^{2}} d y d x \\
= & \int_{I_{0}} \frac{|f(x) \psi(x / a)|^{2}}{|x+a|} d x \leq 2 M\left(\|f\|_{L^{2}\left(I_{0}\right)}\right)^{2}
\end{aligned}
$$




$$
\begin{aligned}
J_{+\infty, 0}=J_{0,+\infty} & =\iint_{I_{0} \times I_{+\infty}} \frac{|f(x) \psi(x / a)|^{2}}{|x-y|^{2}} d y d x \\
& =\int_{I_{0}} \frac{|f(x) \psi(x / a)|^{2}}{|x-a|} d x \leq 2 M\left(\|f\|_{L^{2}\left(I_{0}\right)}\right)^{2} .
\end{aligned}
$$

Summarizing (7.10)-(7.15), we get $\left|\hat{f}_{a}\right|_{0}^{0[1 / 2]}<\infty$, i.e., $\hat{f}_{a} \in H_{0}^{0[1 / 2]}, a>0$. The lemma is proved.

Lemma 7.6. If $f \in H_{0}^{0[1 / 2]}$ is odd, then $\int_{0}^{\infty} \frac{\left|f_{0}(x)\right|^{2}}{x} d x<\infty$.

P r o o f. According to Lemma 7.2, we have $\operatorname{sgn} x f \in H_{0}^{0[1 / 2]}$. Therefore, $H(x) f \in H_{0}^{0[1 / 2]}$, where $H$ is the Heaviside function. Hence,

$$
\int_{0}^{\infty} \frac{|f(x)|^{2}}{x} d x=\int_{0}^{\infty} \int_{0}^{\infty} \frac{|f(x)|^{2}}{|x+y|^{2}} d y d x<\infty
$$

because

$$
2 \int_{0}^{\infty} \int_{0}^{\infty} \frac{|f(x)|^{2}}{|x+y|^{2}} d y d x+\int_{0}^{\infty} \int_{0}^{\infty} \frac{|f(x)-f(y)|^{2}}{|x-y|^{2}}=\left(|H(x) f|_{0}^{0[1 / 2]}\right)^{2}<\infty .
$$

The lemma is proved.

Lemma 7.7. Let $\psi \in C^{2}(\mathbb{R})$ be even; $0 \leq \psi(x) \leq 1, x \in \mathbb{R} ; \psi(x)=1$, $|x| \leq 1 / 2 ; \psi(x)=0,|x| \geq 1$. Let $f \in \widehat{H}_{0}^{1[1 / 2]}, f_{a}(x)=H(x) f(x)(1-\psi(x / a))$, $x \in \mathbb{R}, a>0$. Then $\left|f_{a}\right|_{0}^{1[1 / 2]} \rightarrow 0$ as $a \rightarrow \infty$.

P r o o f. According to (3.3), we have

$$
\iint_{\mathbb{R}^{2} \backslash[-a, a]^{2}} \frac{|f(x)-f(y)|^{2}}{|x-y|^{2}} d y d x \rightarrow 0 \quad \text { as } a \rightarrow \infty,
$$

because for $a=0$ this integral is equal to $\left(|f|_{0}^{0[1 / 2]}\right)^{2}<\infty$. Set $\hat{f}_{a}(x)=$ $f(x) \psi(2 x / a), x \in \mathbb{R}, a>0$. From Lemma 7.5 it follows that $\hat{f}_{a} \in H_{0}^{0[1 / 2]}$. Hence,

$$
\begin{aligned}
& \frac{4}{3 a} \int_{a / 2}^{a}\left|f_{0}(x)\right|^{2} d x \leq \int_{a / 2}^{\infty} \frac{\left|f_{0}(x)\right|^{2}}{x-a / 4} d x \leq \int_{a / 2}^{\infty} \int_{0}^{a / 4} \frac{\left|f(x)-\hat{f}_{a}(x)\right|^{2}}{|x-y|^{2}} d x \\
& \leq \iint_{\mathbb{R}^{2} \backslash[-a / 2, a / 2]^{2}} \frac{\left|\left(f-\hat{f}_{a}\right)(x)-\left(f-\hat{f}_{a}\right)(y)\right|^{2}}{|x-y|^{2}} d y d x \rightarrow 0 \quad \text { as } a \rightarrow \infty
\end{aligned}
$$


because for $a=0$ the last integral is equal to $\left(\left|f-\hat{f}_{a}\right|_{0}^{0[1 / 2]}\right)^{2}<\infty$.

First, let us estimate $\left|\left[f_{a}\right]\right|_{0}^{0[1 / 2]}$. Set $I_{1}=[-\infty, a / 2], I_{2}=[a / 2, a], I_{3}=$ $[a,+\infty]$. Then

$$
\left(\left|f_{a}\right|_{0}^{0[1 / 2]}\right)^{2}=\sum_{k, l=1}^{3} J_{k l}, \quad \text { where } \quad J_{k l}=\iint_{I_{k} \times I_{l}} \frac{\left|f_{a}(x)-f_{a}(y)\right|^{2}}{|x-y|^{2}} d y d x
$$

We have

$$
\begin{aligned}
& J_{11}=0 \text {, } \\
& J_{22} \leq 2 \iint_{I_{2} \times I_{2}} \frac{|f(x)-f(y)|^{2}}{|x-y|^{2}} d y d x \\
& +2 \iint_{I_{2} \times I_{2}}|f(x)|^{2} \frac{|\psi(x / a)-\psi(y / a)|^{2}}{|x-y|^{2}} d y d x, \\
& J_{33}=\iint_{I_{3} \times I_{3}} \frac{|f(x)-f(y)|^{2}}{|x-y|^{2}} d y d x, \\
& J_{12}=J_{21}=\iint_{I_{2} \times I_{1}}|f(x)|^{2} \frac{|1-\psi(x / a)|^{2}}{|x-y|^{2}} d y d x \\
& \leq \int_{I_{2}}|f(x)|^{2} \frac{|1-\psi(x / a)|^{2}}{x-a / 2} d x, \\
& J_{13}=J_{31}=\iint_{I_{3} \times I_{1}} \frac{|f(x)|^{2}}{|x-y|^{2}} d y d x=\int_{a}^{\infty} \frac{|f(x)|^{2}}{x-a / 2} d x, \\
& J_{23}=J_{32}=2 \iint_{I_{3} \times I_{2}} \frac{|f(x)-f(y)|^{2}}{|x-y|^{2}} d y d x \\
& +2 \iint_{I_{3} \times I_{2}}|f(y)|^{2} \frac{|\psi(y / a)|^{2}}{|x-y|^{2}} d y d x \\
& =2 \iint_{I_{3} \times I_{2}} \frac{|f(x)-f(y)|^{2}}{|x-y|^{2}} d y d x \\
& +2 \int_{I_{2}}|f(y)|^{2} \frac{|\psi(y / a)|^{2}}{|a-y|^{2}} d y \text {. }
\end{aligned}
$$

Applying the mean value theorem, we obtain $|\psi(x / a)-\psi(y / a)| \leq \frac{M}{a}|x-y|$, $(x, y) \in \mathbb{R}^{2}$, where $M=\max \left\{\left|\psi^{\prime}(\xi)\right||| \xi \mid \leq 1\right\}$. Therefore,

$$
\iint_{I_{2} \times I_{2}}|f(x)|^{2} \frac{|\psi(x / a)-\psi(y / a)|^{2}}{|x-y|^{2}} d y d x \leq \frac{M^{2}}{2 a} \int_{a / 2}^{a}|f(x)|^{2} d x
$$




$$
\begin{aligned}
\int_{I_{2}}|f(x)|^{2} \frac{|1-\psi(x / a)|^{2}}{x-a / 2} d x & \leq \frac{M^{2}}{a^{2}} \int_{a / 2}^{a}|f(x)|^{2}(x-a / 2) d x \\
& \leq \frac{M^{2}}{2 a} \int_{a / 2}^{a}|f(x)|^{2} d x \\
\int_{I_{2}}|f(y)|^{2} \frac{|\psi(y / a)|^{2}}{|a-y|^{2}} d y & \leq \frac{M^{2}}{a^{2}} \int_{a / 2}^{a}|f(y)|^{2}(a-y) d y \\
& \leq \frac{M^{2}}{2 a} \int_{a / 2}^{a}|f(y)|^{2} d y .
\end{aligned}
$$

Summarizing (7.16)-(7.27), we get

$$
\begin{aligned}
\left|f_{a}\right|_{0}^{0[1 / 2]} \leq & \left(\iint_{\mathbb{R}^{2} \backslash[-a / 2, a / 2]^{2}} \frac{|f(x)-f(y)|^{2}}{|x-y|^{2}} d y d x+2 \int_{a}^{\infty} \frac{|f(x)|^{2}}{x-a / 2} d x\right. \\
& \left.+\frac{3 M^{2}}{a} \int_{a / 2}^{a}|f(x)|^{2} d x\right)^{1 / 2} \rightarrow 0 \quad \text { as } a \rightarrow \infty .
\end{aligned}
$$

Analogously, taking into account Lemma 7.6 and applying the assertion

$$
\frac{1}{a} \int_{a / 2}^{a}\left|f^{\prime}(x)\right| d x \leq \int_{a / 2}^{\infty} \frac{\left|f^{\prime}(x)\right|^{2}}{x} d x \rightarrow 0 \quad \text { as } a \rightarrow \infty
$$

instead of (7.17), we obtain that $\left|\left(f_{a}\right)^{\prime}\right|_{0}^{1[1 / 2]} \rightarrow 0$ as $a \rightarrow \infty$. Thus, $\left|f_{a}\right|_{0}^{1[1 / 2]} \rightarrow 0$ as $a \rightarrow \infty$. That was to be proved.

Lemma 7.8. Let $u \in L^{\infty}\left(\mathbb{R}_{+}\right), p(x, t)=\frac{\partial}{\partial x_{1}} \int_{0}^{t} \frac{H\left(\xi^{2}-|x|^{2}\right)}{\sqrt{\xi^{2}-|x|^{2}}} u(t-\xi) d \xi$, and $p^{+}$ be the restriction of $p$ to $\mathbb{R}_{+} \times \mathbb{R} \times \mathbb{R}_{+}$. Then

$$
p^{+}\left(0, x_{2}, t\right)=\pi \delta\left(x_{2}\right) u(t)
$$

P r o o f. One can see that

$$
p(x, t)=-\frac{x_{1}}{|x|^{2}}\left(\frac{\partial}{\partial t}\right)^{2} \int_{0}^{t} H\left(\xi^{2}-|x|^{2}\right) \sqrt{\xi^{2}-|x|^{2}} u(t-\xi) d \xi .
$$

Let $\alpha>0$ and $\psi \in \mathcal{S}_{+} \times \mathcal{S} \times \mathcal{S}^{+}$. For $x \in \mathbb{R}^{2}, \xi \in \mathbb{R}_{+}$, and $t \in \mathbb{R}_{+}$, set

$$
\begin{aligned}
f_{\alpha}(x, \xi) & =\frac{\alpha x_{1}}{\left(\alpha x_{1}\right)^{2}+x_{2}^{2}} H\left(\xi^{2}-\left(\alpha x_{1}\right)^{2}-x_{2}^{2}\right) \sqrt{\xi^{2}-\left(\alpha x_{1}\right)^{2}-x_{2}^{2}}-\pi \delta\left(x_{2}\right), \\
g_{\alpha}(x, t) & =\left(\frac{\partial}{\partial t}\right)^{2} \int_{0}^{t} u(t-\xi) f_{\alpha}(x, \xi) d \xi \\
\varphi(x, \xi) & =\int_{\xi}^{\infty} \psi_{t t}(x, t) u(t-\xi) d t .
\end{aligned}
$$


Therefore, (7.28) is equivalent to

$$
\left\langle f_{\alpha}, \varphi\right\rangle=\left\langle g_{\alpha}, \psi\right\rangle \rightarrow 0 \text { as } \alpha \rightarrow+0 .
$$

We have $\left\langle f_{\alpha}, \varphi\right\rangle=\sum_{j=1}^{4}\left\langle f_{\alpha}^{j}, \varphi\right\rangle$, where

$$
\begin{aligned}
& f_{\alpha}^{1}(x, \xi)=\frac{\alpha x_{1} H\left(\xi^{2}-\left(\alpha x_{1}\right)^{2}-x_{2}^{2}\right)}{\left(\alpha x_{1}\right)^{2}+x_{2}^{2}}\left(\sqrt{\xi^{2}-\left(\alpha x_{1}\right)^{2}-x_{2}^{2}}-\sqrt{\xi^{2}-\left(\alpha x_{1}\right)^{2}}\right), \\
& f_{\alpha}^{2}(x, \xi)=\frac{\alpha x_{1} \sqrt{\xi^{2}-\left(\alpha x_{1}\right)^{2}}}{\left(\alpha x_{1}\right)^{2}+x_{2}^{2}}\left(H\left(\xi^{2}-\left(\alpha x_{1}\right)^{2}-x_{2}^{2}\right)-H\left(\xi^{2}-\left(\alpha x_{1}\right)^{2}\right)\right), \\
& f_{\alpha}^{3}(x, \xi)=\sqrt{\xi^{2}-\left(\alpha x_{1}\right)^{2}} H\left(\xi^{2}-\left(\alpha x_{1}\right)^{2}\right)\left(\frac{\alpha x_{1}}{\left(\alpha x_{1}\right)^{2}+x_{2}^{2}}-\pi \delta\left(x_{2}\right)\right), \\
& f_{\alpha}^{4}(x, \xi)=\pi \delta\left(x_{2}\right)\left(\sqrt{\xi^{2}-\left(\alpha x_{1}\right)^{2}} H\left(\xi^{2}-\left(\alpha x_{1}\right)^{2}\right)-|\xi|\right), \quad(x, \xi) \in \mathbb{R}^{2} \times \mathbb{R}_{+} .
\end{aligned}
$$

Since $\psi \in \mathcal{S}^{+} \times \mathcal{S} \times \mathcal{S}^{+}$, we get $\varphi \in \mathcal{S}^{+} \times \mathcal{S} \times \mathcal{S}^{+}$and

$$
|\varphi(x, \xi)| \leq \frac{M}{\left(1+x_{1}^{2}\right)^{2}\left(1+\xi^{2}\right)}, \quad(x, \xi) \in \mathbb{R}^{2} \times \mathbb{R}_{+},
$$

where $M>0$. Applying the mean value theorem, we also obtain

$$
\left|\varphi\left(x_{1}, x_{2}, \xi\right)-\varphi\left(x_{1}, 0, \xi\right)\right| \leq \frac{C_{s p}^{k l}\left|x_{1}\right|^{k}\left|x_{2}\right|^{l}}{\left(1+x_{1}^{2}\right)^{s}\left(1+\xi^{2}\right)^{p}}, \quad(x, \xi) \in \mathbb{R}^{2} \times \mathbb{R}_{+},
$$

where $C_{s p}^{k l}>0, k=0,1, l=0,1, s=\overline{0, \infty}, l=\overline{0, \infty}$, because $\varphi\left(0, x_{2}, \xi\right)=0$ for $x_{2} \in \mathbb{R}$ and $\xi \in \mathbb{R}_{+}$.

Now, let us estimate $\left\langle f_{\alpha}^{j}, \varphi\right\rangle, j=\overline{1,4}$. Taking into account (7.30), we obtain

$$
\begin{aligned}
\left|\left\langle f_{\alpha}^{1}, \varphi\right\rangle\right| & \leq \alpha \iiint_{\mathbb{R}^{3}} \frac{H\left(\xi^{2}-\left(\alpha x_{1}\right)^{2}-x_{2}^{2}\right) x_{2}^{2}\left|x_{1} \varphi(x, \xi)\right|}{\sqrt{\xi^{2}-\left(\alpha x_{1}\right)^{2}-x_{2}^{2}}\left(\left(\alpha x_{1}\right)^{2}+x_{2}^{2}\right)} d x d \xi \\
& \leq 2 M \pi \alpha \int_{0}^{\infty} \frac{x_{1} d x_{1}}{\left(1+x_{1}^{2}\right)^{2}} \int_{0}^{\infty} \frac{d \xi}{1+\xi^{2}}=M \pi^{2} \alpha \\
\left|\left\langle f_{\alpha}^{2}, \varphi\right\rangle\right| & \leq 2 M \iint_{\mathbb{R}^{2}} \frac{\alpha\left|x_{1}\right| \sqrt{\xi^{2}-\left(\alpha x_{1}\right)^{2}}}{\left(1+x_{1}^{2}\right)\left(1+\xi^{2}\right)} \int_{0}^{\infty} \frac{H\left(\xi^{2}-\left(\alpha x_{1}\right)^{2}-x_{2}^{2}\right) d x_{2}}{\left(\alpha x_{1}\right)^{2}+x_{2}^{2}} d x_{1} d \xi \\
& \leq 2 M \alpha \int_{0}^{\infty} \frac{x_{1} d x_{1}}{\left(1+x_{1}^{2}\right)^{2}} \int_{0}^{\infty} \frac{d \xi}{1+\xi^{2}}=M \pi \alpha \leq M \pi^{2} \alpha
\end{aligned}
$$

In (7.33) we have used the inequality $\left|\frac{\pi}{2}-\arctan \frac{1}{z}\right| \leq z, z>0$. Applying again this inequality and (7.31), we obtain

$$
\left|\left\langle f_{\alpha}^{3}, \varphi\right\rangle\right| \leq \iiint_{\mathbb{R}^{3}} \frac{\alpha\left|\xi x_{1}\right|}{\left(\alpha x_{1}\right)^{2}+x_{2}^{2}}\left|\varphi(x, \xi)-\varphi\left(x_{1}, 0, \xi\right)\right| d x_{1} d \xi
$$




$$
\begin{aligned}
& \leq 2 \iint_{\mathbb{R}^{2}} \frac{\alpha\left|\xi x_{1}\right|}{\left(1+\xi^{2}\right)^{2}}\left(\frac{C_{12}^{11}\left|x_{1}\right|}{1+x_{1}^{2}} \int_{0}^{\alpha^{3 / 4}} \frac{x_{2} d x_{2}}{\left(\alpha x_{1}\right)^{2}+x_{2}^{2}}\right. \\
& \left.\quad+\frac{C_{22}^{00}}{\left(1+x_{1}^{2}\right)^{2}} \int_{\alpha^{3 / 4}}^{\infty} \frac{d x_{2}}{\left(\alpha x_{1}\right)^{2}+x_{2}^{2}}\right) d x_{1} d \xi \\
& =\iint_{\mathbb{R}^{2}} \frac{|\xi|}{\left(1+\xi^{2}\right)^{2}}\left(\frac{\alpha C_{12}^{11} x_{1}^{2}}{1+x_{1}^{2}} \ln \frac{\left(\alpha x_{1}\right)^{2}+\alpha^{3 / 2}}{\left(\alpha x_{1}\right)^{2}}\right. \\
& \left.\quad+\frac{2 C_{22}^{00}}{\left(1+x_{1}^{2}\right)^{2}}\left(\frac{\pi}{2}-\arctan \frac{\alpha^{3 / 4}}{\alpha\left|x_{1}\right|}\right)\right) d x_{1} d \xi \\
& \leq\left(C_{12}^{11} \alpha^{1 / 2}+C_{22}^{00} \alpha^{1 / 4}\right) \int_{0}^{\infty} \frac{d x_{1}}{1+x_{1}^{2}} \int_{0}^{\infty} \frac{\xi d \xi}{\left(1+\xi^{2}\right)^{2}} \\
& =2 \pi\left(C_{12}^{11} \alpha^{1 / 2}+C_{22}^{00} \alpha^{1 / 4}\right) .
\end{aligned}
$$

Taking into account (7.30), we get

$$
\begin{aligned}
\left|\left\langle f_{\alpha}^{3}, \varphi\right\rangle\right| \leq 4 \pi \int_{0}^{\infty}\left(\int_{0}^{\alpha x_{1}}\left|\xi \varphi\left(x_{1}, 0 \xi\right)\right| d x_{1}\right. \\
\left.\quad+\int_{\alpha x_{1}}^{\infty}\left(|\xi|-\sqrt{\xi^{2}-\left(\alpha x_{1}\right)^{2}}\right)\left|\varphi\left(x_{1}, 0, \xi\right)\right| d x_{1}\right) d \xi \\
\leq 4 \pi \alpha \int_{0}^{\infty} \int_{0}^{\infty} x_{1}\left|\varphi\left(x_{1}, 0, \xi\right)\right| d x_{1} d \xi \\
\leq 4 M \pi \alpha \int_{0}^{\infty} \frac{x_{1} d x_{1}}{\left(1+x_{1}^{2}\right)^{2}} \int_{0}^{\infty} \frac{d \xi}{1+\xi^{2}}=2 M \pi^{2} \alpha .
\end{aligned}
$$

Summarizing (7.32)-(7.35), we obtain

$$
\left|\left\langle f_{\alpha}, \varphi\right\rangle\right| \leq 7 M \pi^{2} \alpha+2 \pi\left(C_{12}^{11} \alpha^{1 / 2}+C_{22}^{00} \alpha^{1 / 4}\right) \rightarrow 0 \quad \text { as } \alpha \rightarrow+0 .
$$

Thus (7.29) holds for each $\psi \in \mathcal{S}_{+} \times \mathcal{S} \times \mathcal{S}^{+}$. That was to be proved.

Lemma 7.9. Let $g \in \widehat{H}_{0}^{0}\left(\mathbb{R}^{2}\right), f(x, t)=\frac{\partial}{\partial x_{1}} \frac{H\left(t^{2}-|x|^{2}\right)}{\sqrt{t^{2}-|x|^{2}}} * g$, and $f^{+}$be the restriction of $f$ to $\mathbb{R}_{+} \times \mathbb{R} \times \mathbb{R}_{+}$. Here $*$ is the convolution with respect to $x$. Then

$$
f\left(0, x_{2}, t\right)=0 .
$$

P r o o f. Let $\alpha>0, \psi \in \mathcal{S}_{+} \times \mathcal{S} \times \mathcal{S}^{+}$, and $\widehat{\psi}(x, t)=\psi\left(x_{1}, x_{2}, t\right)-\psi\left(-x_{1}, x_{2}, t\right)$, $x \in \mathbb{R}^{2}$ and $t \in \mathbb{R}_{+}$. Set $\Psi(\cdot, t)=\mathcal{F}_{x \rightarrow \sigma} \psi(\cdot, t), G=\mathcal{F} g, P(\sigma, t)=\frac{\sigma_{1}}{|\sigma|} \sin (t|\sigma|)$, $\Psi_{\alpha}(\sigma, t)=\Psi\left(\alpha \sigma_{1}, \sigma_{2}, t\right)$, and $f_{\alpha}(x, t)=f\left(\alpha x_{1}, x_{2}, t\right)$, where $\sigma \in \mathbb{R}^{2}, x \in \mathbb{R}^{2}$, $t \in \mathbb{R}_{+}$. Then, $\left\langle F_{\alpha}, \psi\right\rangle=\left\langle P G, \Psi_{\alpha}\right\rangle$. Therefore, to prove (7.36), we have to prove

$$
\left\langle P G, \Psi_{\alpha}\right\rangle \rightarrow 0 \quad \text { as } \alpha \rightarrow+0 .
$$


Since $P(\sigma, t)=\frac{\partial}{\partial t}\left(\frac{\sigma_{1}}{|\sigma|^{2}}(1-\cos (t|\sigma|))\right)$, we get

$$
\left\langle P G, \Psi_{\alpha}\right\rangle=-\frac{1}{2} \int_{0}^{\infty} \iint_{\mathbb{R}^{2}} \frac{\sigma_{1}}{|\sigma|^{2}}(1-\cos (t|\sigma|)) G(\sigma) \Psi_{t}\left(\alpha \sigma_{1}, \sigma_{2}, t\right) d \sigma d t .
$$

We have

$$
\left|\Psi_{t}\left(\sigma_{1}, \sigma_{2}, t\right)\right| \leq \frac{M^{k}|\sigma|^{k}}{\left(1+\sigma_{2}^{2}\right)^{1 / 2}\left(1+t^{2}\right)}, \quad(\sigma, t) \in \mathbb{R}^{2} \times \mathbb{R}_{+},
$$

where $M^{k}>0, k=0,1$, because $\Psi_{t} \in \mathcal{S} \times \mathcal{S} \times \mathcal{S}^{+}$and $\psi_{t}\left(0, \sigma_{2}, t\right)=0,\left(\sigma_{2}, t\right) \in$ $\mathbb{R} \times \mathbb{R}_{+}$. Hence,

$$
\begin{aligned}
\left|\left\langle P G, \Psi_{\alpha}\right\rangle\right| \leq 2 \int_{0}^{\infty} \frac{d t}{1+t^{2}} \int_{0}^{\infty} \frac{1}{\sqrt{1+\sigma_{2}^{2}}}\left(\alpha M^{1} \int_{0}^{1 / \alpha} \frac{\sigma_{1}^{2}|G(\sigma)|}{\sigma_{1}^{2}+\sigma_{2}^{2}} d \sigma_{1}\right. \\
\left.+M^{0} \int_{1 / \alpha}^{\infty} \frac{\sigma_{1}|G(\sigma)|}{\sigma_{1}^{2}+\sigma_{2}^{2}} d \sigma_{1}\right) d \sigma_{2} \\
\leq \pi \int_{0}^{\infty} \frac{1}{\sqrt{1+\sigma_{2}^{2}}} \sqrt{\int_{0}^{\infty}\left|G\left(\sigma_{1}, \sigma_{2}\right)\right|^{2} d \sigma_{1}}\left(\alpha M^{1} \sqrt{\int_{0}^{1 / \alpha} d \sigma_{1}}\right. \\
+M^{0} \sqrt{\left.\int_{1 / \alpha}^{\infty} \frac{d \sigma_{1}}{\sigma_{1}^{2}}\right) d \sigma_{2}} \\
\leq\left(M^{0}+M^{1}\right) \sqrt{\frac{\pi^{3} \alpha}{2}}\|g\|_{0}^{0} \rightarrow 0 \quad \text { as } \alpha \rightarrow+0 .
\end{aligned}
$$

That was to be proved.

\section{References}

[1] P. Antosik, J. Mikusiński, and R. Sikorski, Theory of Distributions. The Sequential Approach, Elsevier, Amsterdam, 1973.

[2] M.I. Belishev and A.F. Vakulenko, On a Control Problem for the Wave Equation in $\mathbb{R}^{3}$. - Zapiski Nauchnykh Seminarov POMI 332 (2006), 19-37. (Russian). (Engl. transl.: J. Math. Sci. 142 (2007), 2528-2539.)

[3] L.V. Fardigola, On Controllability Problems for the Wave Equation on a Half-Plane. - J. Math. Phys., Anal., Geom. 1 (2005), 93-115.

[4] L.V. Fardigola, Controllability Problems for the String Equation on a Half-Axis with a Boundary Control Bounded by a Hard Constant. — SIAM J. Control Optim. 47 (2008), 2179-2199. 
[5] L.V. Fardigola, Neumann Boundary Control Problem for the String Equation on a Half-Axis. — Dopovidi Natsionalnoi Akademii Nauk Ukrainy (2009), No. 10, 36-41. (Ukrainian)

[6] L.V. Fardigola, Controllability Problems for the 1-d Wave Equation on a Half-Axis with the Dirichlet Boundary Control. — ESAIM: Control, Optim. Calc. Var. 18 (2012), 748-773.

[7] L.V. Fardigola, Transformation Operators of the Sturm-Liouville Problem in Controllability Problems for the Wave Equation on a Half-Axis. — SIAM J. Control Optim. 51 (2013), 1781-1801.

[8] L.V. Fardigola and K.S. Khalina, Controllability Problems for the Wave Equation. — Ukr. Mat. Zh. 59 (2007), 939-952. (Ukrainian). (Engl. transl.: Ukr. Math. J. 59 (2007), 1040-1058.)

[9] I.M. Gelfand and G.E. Shilov, Generalized Functions. Vol. 2. Fismatgiz, Moscow, 1958. (Russian)

[10] S.G. Gindikin and L.R. Volevich, Distributions and Convolution Equations. Gordon and Breach, Philadelphia, 1992.

[11] M. Gugat, Optimal Switching Boundary Control of a String to Rest in Finite Time. — ZAMM Angew. Math. Mech. 88 (2008), 283-305.

[12] M. Gugat and G. Leugering, $L^{\infty}$-norm Minimal Control of the Wave Equation: on the Weakness of the Bang-Bang Principle. - ESAIM: Control, Optim. Calc. Var. 14 (2008), 254-283.

[13] M. Gugat, G. Leugering, and G. Sklyar, $L^{p}$-optimal Boundary Control for the Wave Equation. - SIAM J. Control Optim. 44 (2005), 49-74.

[14] V.A. Il'in and E.I. Moiseev, A Boundary Control at Two Ends by a Process Described by the Telegraph Equation. - Dokl. Akad. Nauk, Ross. Akad. Nauk 394 (2004) No. 2, 154-158. (Russian). (Engl. transl.: Doklady Mathematics, 69 (2004) No. 1, 33-37.)

[15] K.S. Khalina Boundary Control Problems for the Equation of Vibrating of Nonhomogeneous String on a Half-Axis. - Ukr. Mat. Zh. 64 (2012) No. 4, 525-541.

[16] K.S. Khalina On the Neumann Boundary Controllability for the Non-homogeneous String on a Half-Axis. — J. Math. Phys., Anal., Geom. 8 (2012) No. 4, 307-335.

[17] G.M. Sklyar and L.V. Fardigola, The Markov Power Moment Problem in Problems of Controllability and Frequency Extinguishing for the Wave Equation on a HalfAxis. - J. Math. Anal. Appl. 276 (2002), 109-134.

[18] J. Vancostenoble and E. Zuazua, Hardy Inequalities, Observability, and Control for the Wave and Schrödinger Equations with Singular Potentials. — SIAM J. Math. Anal. 41 (2009), 1508-1532.

[19] V.S. Vladimirov, Equations of Mathematical Physics. Mir, Moscow, 1984. 